\title{
On the detection of natural frequencies and mode shapes of submerged rotating disk-like structures from the casing
}

\author{
Alexandre Presas ${ }^{a, *}$, David Valentin ${ }^{a}$, Eduard Egusquiza ${ }^{a}$, \\ Carme Valero ${ }^{\mathrm{a}}$, Ulrich Seidel ${ }^{\mathrm{b}}$ \\ a Centre de Diagnòstic Industrial i Fluidodinàmica, Universitat Politècnica de Catalunya, Av. Diagonal, 647, ETSEIB, Pab. D+1, \\ 08028 Barcelona, Spain \\ ${ }^{\mathrm{b}}$ Voith Hydro Holding GmbH E' Co. KG, Alexanderstraße 11, 89522 Heidenheim, Germany
}

\section{A R T I C L E I N F O}

\section{Article history:}

Received 25 July 2014

Received in revised form

5 November 2014

Accepted 14 January 2015

\section{Keywords:}

Rotating disk

Submerged structure

Natural frequencies detection

PZT

Hydraulic machinery

\begin{abstract}
A B S T R A C T
To avoid resonance problems in rotating turbomachinery components such as impellers, it is of paramount importance to determine the natural frequencies of these parts when they are under operation. Nevertheless, most of these rotating structures are inaccessible and in some cases submerged and confined. To measure the natural frequencies of submerged impellers from the rotating frame is complicated, because sensors have to be well fixed, withstand with large pressure and centrifugal forces. Furthermore, the signals have to be transmitted to the stationary frame. For this reason it may be advantageous to measure the natural frequencies with sensors placed on the casing.

In this paper, the analysis of rotating disk-like structures submerged and confined has been performed from the stationary frame. Previously, an analytical model to determine the natural frequencies and mode shapes of the disk from the rotating frame is presented. Once natural frequencies and mode shapes are obtained in the rotating frame, the transmission to the stationary frame has been deduced.

A rotating disk test rig has been used for the experimental study. It consist of a rotating disk that has been excited from the rotating frame with a piezoelectric patch and it response has been measured from both rotating and stationary frame. Results shows that for rotating submerged structures in heavy fluids such as water, not only the structural modes of the rotating part are different than for rotating structures in air, but also the transmission from the rotating to the stationary frame.
\end{abstract}

(c) 2015 Elsevier Ltd. All rights reserved.

\section{Introduction}

The vibration of rotating disk-like structures has been studied extensively in the last years due to their relevance in real engineering applications such as circular saws, cutters, hard disks or turbomachinery components. Particularly in hydraulic turbomachinery, some types of runners are disk-like structures which are submerged and confined rotating in water. When they are in operation the interaction between the stationary guide vanes and rotating blades produces an excitation (rotor stator interaction) that may causes resonance problems which lead to fatigue damages or critical failures on the rotating

\footnotetext{
* Corresponding author. Tel.: +34 93 4016715; fax: +34 934015812

E-mail address: alex.presas@mf.upc.edu (A. Presas).

URL: http://www.upc.edu/cdif (A. Presas).
} 
structure [1-5]. The similarity between the mode shapes of runners and disks, especially for the first modes of vibration, has been commented in many studies [1-10]. Particularly, the first modes of some kind of hydraulic runners are characterized by the number of nodal diameters, which characterize also the mode shapes of a simple disk.

Many studies deal with the topic of rotating disk-like structures, rotating in air [11-18]. The effect of rotation in the dynamic behavior of the disk was first studied by Lamb and Southwell [11]. Campbell [12] introduced the term critical speed, at which a standing wave appears on the disk. Southwell [13] studied the vibration of circular disks clamped at its center, which is in practice, the most used configuration. Later researches, studied more complex structures such as bladed disks [14,15]. In [14] Ewins compared the stress levels of a perfect bladed disk and a mistuned bladed disk under operation. El-Bayoumy and Srinivasan [15] studied the stress levels of turbine and compressor blades of mistuned bladed structures. Newer studies [16-18] determined other effects using numerical simulation. In [16] Heo and Chung studied the effect of misalignment in the natural frequencies of the disk. Bauer and Eidel [17] studied the effect of the attachment to the stationary part. Pust and Pesek [18] studied a bladed disk with imperfections. All these researches give a good knowledge of different effects that are relevant for the dynamic response of rotating disks, nevertheless these studies did not consider the interaction of the disk with the surrounding media.

The surrounding fluid has a relevant effect on the natural frequencies of the structure if its density is high, because the added mass effect. The added mass effect of water in static structures that are surrounded by water has been studied in many cases [19-22]. Lamb [19] studied the added mass effect of water in the vibration of a thin plate. Recently, Kwak in [20] and Amabili and Kwak in [21] reviewed the problem of Lamb, emphasizing the added virtual mass incremental factors (AVMI) for each mode of the disk. Some studies have been also performed where the disk is totally confined, for example [22,23]. Although the added mass effect of water is well characterized in these studies for static structures, the effect of a rotating structure that induces a relative motion between the structure and the surrounding fluid is not studied in these cases.

The study of rotating disks submerged in water or water that rotates with respect to the disk has been considered in few cases $[3,24,25]$. Kubota and Ohashi [3] provided an analytical model for a stationary disk and water that rotates with respect to the disk. Nevertheless, no experimental results were given. Hengstler [24] provide experimental results for a submerged and confined disk, with rotating water on the lower surface. In both cases, the water was rotating with respect to the disk, but the disk was standing. Only Presas et al. in [25] studied a disk that is forced to rotate inside a tank full of water. Nevertheless the analysis of the natural frequencies was performed from the rotating frame.

Analyzing the problem from the stationary frame can be advantageous in case of real turbomachines. In this case, rotating parts are usually inaccessible and in hydraulic turbomachinery, also submerged. Furthermore, sensors installed on the rotating frame have to withstand with high centrifugal forces and pressure fluctuations and the signals have to be transmitted to the stationary frame. For all these reasons, it could be easier to install sensors on the casing (stationary frame) to detect natural frequencies and mode shapes of the rotating frame. Nevertheless, the frequency content of the signals acquired from the stationary frame is more difficult to be interpreted, due to the difference within measured (rotating) and instrumentation frame (stationary).

For disks that rotate in air, the correlation between natural frequencies in the rotating frame and in the stationary frame is well known. This relation depends on the rotating speed of the impeller and also on the mode shape as deduced mathematically and proven experimentally in [26,27]. Nevertheless, no experimental or analytical studies have been found with the measurement of the natural frequencies of submerged rotating disks in water from the stationary frame (casing).

In this paper the detection of the natural frequencies and mode shapes of a submerged rotating disk-like structure from the stationary frame is studied in detail. First, this transmission is studied analytically. The natural frequencies of a submerged and rotating disk are determined from the stationary reference frame. To analyze the transmission experimentally a rotating disk test rig has been used. It consists of a disk that is excited from the rotating frame with a piezoelectric patch and its response measured from the rotating and stationary frame simultaneously. Therefore, measurements on the rotating and on the stationary frame have been compared and all the conclusions of the analytical model have been validated with experimental results.

\section{Analytical model}

\subsection{Natural frequencies and mode shapes of a rotating disk considering the surrounding fluid}

The model developed by Kubota [3] is used to study the natural frequencies and mode shapes of a rotating disk considering the surrounding fluid. In this paper, this model has been particularized for a totally confined disk (Fig. 1).

As shown in Fig. 1 cylindrical coordinates are used to describe the problem. The disk rotates at a constant speed $\Omega_{\text {disk }}$ with respect to the stationary frame. This induces a motion of the surrounding fluid, which has an average speed $\Omega_{\text {fluid }}$, with respect to the stationary frame. Both are positive definite in the direction shown in Fig. 1. Therefore the following relations can be deduced for the angular coordinate $\theta$ :

$$
\left\{\begin{array}{c}
\theta_{\text {disk }}=\theta_{\text {casing }}+\Omega_{\text {disk }} \times t \\
\theta_{\text {fluid-up }}=\theta_{\text {casing }}+\Omega_{\text {fluid-up }} \times t \\
\theta_{\text {fluid-down }}=\theta_{\text {casing }}+\Omega_{\text {fluid-down }} \times t \\
\Omega_{\text {fluid/disk }}=\Omega_{\text {disk }}-\Omega_{\text {fluid }}
\end{array}\right.
$$




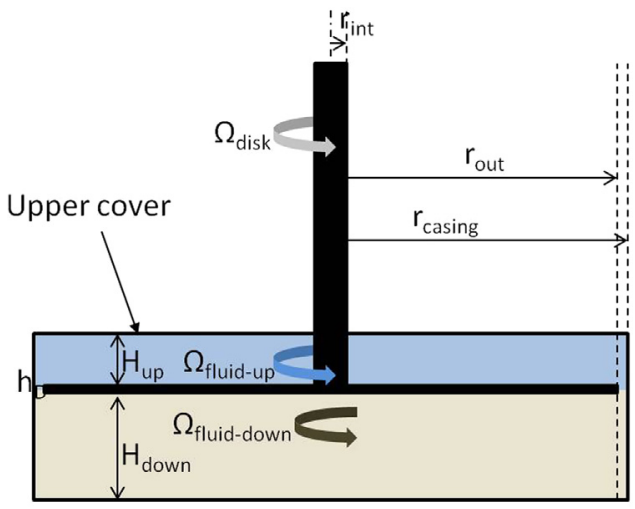

Fig. 1. Rotating disk confined with surrounding fluid.

The transverse free vibration of a rotating disk in vacuum can be expressed as [11]

$$
\rho_{D} h_{D} \frac{\partial^{2} w}{\partial t^{2}}+D\left[\frac{\partial}{\partial r^{2}}+\frac{1}{r} \frac{\partial}{\partial r}+\frac{1}{r^{2}} \frac{\partial^{2}}{\partial \theta^{2}}\right]^{2} w=0
$$

In Eq. (2), $\rho_{D}$ and $h_{D}$ are the density and thickness of the disk, respectively. $w$ is the transverse displacement of the disk. $D$ is the bending stiffness $\left(D=\left(E h_{D}^{3}\right) /\left(12\left(1-v^{2}\right)\right)\right)$ with $E$ and $v$ the Young and Poisson modulus of the material.

With the boundary conditions of the disk (clamped at the center and free at the edge) the solution of Eq. (2) can be expressed as [28]

$$
w_{\text {disk }}=w_{\text {disk }}(r, \theta, t)=\sum_{n=0}^{\infty} \sum_{m=0}^{\infty} W_{n m}(r) \cos (n \theta) e^{j \omega_{n m} t}
$$

In Eq. (3), the solution is expressed as the superposition of free vibrating modes that are characterized by $n$ and $m$. $n$ is the number of nodal diameters of the mode and $m$ the number of nodal circles. $\omega_{n m}$ is the corresponding natural frequency of the mode.

Only the modes with no nodal circles, i.e. characterized by the number of nodal diameters will be discussed here (diametrical modes). According to many studies [1,3,6-10], the diametrical modes of some types of hydraulic impellers are the most prompt to be excited during the operation of these machines. Diametrical modes are also characteristic modes in a simple disk.

For certain kind of annular disks and considering only the diametrical modes with $n>1$, it can be demonstrated that the motion of the disk can be simplified assuming that the vibration of the disk is uniform in the radial direction and considering the vibration in an averaged radius $r_{o}=\sqrt{r_{i n} \times r_{\text {out }}}$. In this case the surrounding fluid is considered taking into account the pressure that the fluid is applying on the disk at $r=r_{o}$ [3].

$$
\rho_{D} h_{D} \frac{\partial^{2} w}{\partial t^{2}}+\frac{D^{*}}{r_{o}^{4}} \frac{\partial^{4} w}{\partial \theta^{4}}=p_{r_{o}}
$$

In this equation, $D^{*}$ is a parameter that depends on structural properties of the disk. For the considered mode shapes, the disk vibration at $r_{o}$ can be expressed as

$$
w_{\text {disk }}=\sum_{n= \pm 2}^{\infty} A_{n} e^{j n \theta} e^{j \omega_{n} t}
$$

where $|n|$ indicates the number of nodal diameters in the mode shape and the sign of $\mathrm{n}$ indicates the direction of the travelling wave that appears on the disk. $\omega_{n}$ is the corresponding natural frequency.

To calibrate $D^{*}$ and to know the validity of the mentioned assumptions the following procedure is performed. Considering the case that the disk is vibrating in vacuum $\left(p_{r_{o}}=0\right.$ in Eq. (4)) and combining Eq. (5) with Eq. (4) the natural frequencies for each mode $\mathrm{n}$ can be calculated as

$$
\omega_{n, \text { vacuum }}^{2}=\frac{n^{4} D^{*}}{\rho_{D} h_{D} r_{o}^{4}}
$$

Comparing the results obtained with Eq. (6) and the results found in $[28,29], D^{*}$ can be calibrated and the error of the approach calculated. It can be demonstrated that the use of Eq. (4) (with $p_{r_{0}}=0$ ) is acceptable for modes $n>1$ and for thin disks, with small inner radius compared to outer radius.

To consider the surrounding fluid in the vibration of the disk, $p_{r_{o}}$ has to be calculated. It is assumed that the surrounding fluid of the disk can be described by a potential flow [30]. Therefore, this flow is considered as an irrotational, inviscid and 
incompressible flow. Two fluid fields are considered here, the upper fluid and the lower fluid (Fig. 1). The velocity potentials of both fluid fields are $U_{u p}$ and $U_{\text {down }}$, respectively. Both are considered to rotate at a constant speed $\Omega_{\text {fluid-up }}$ and $\Omega_{\text {fluid-down }}$ with respect to the disk. This rotation of the fluid with respect to the disk is induced by the rotation of the disk (that is connected to a motor). In the real case, since the fluid is viscous, the flow pattern has not a constant rotating speed and therefore an averaged rotating speed has to be calculated. In this study $\Omega_{\text {fluid-up }}$ and $\Omega_{\text {fluid-down }}$ have been obtained by means of a CFD simulation of the flow inside the tank when the disk is rotating, without considering the transverse vibration of the disk.

For the CFD simulation, Navier-Stokes Equations have been solved numerically using Ansys Fluent ${ }^{\circledR}$ v14.5 software. A pressure based-double precision solver has been selected in order to solve the set of Equations used. Second order upwind discretization schemes have been imposed on the equations solved (momentum, energy and turbulence). Simulations are performed in steady state. A 3D structured hexahedral grid has been applied to the geometrical model. Only the fluid field has been considered, fixing the nodes in contact with the casing as a standing wall and imposing a rotation speed in the nodes corresponding to the disk. A mesh sensitivity study has been performed to ensure the model is adequately accurate. In this case, the mean velocity in a constant radius of the disc $\left(r_{o}\right)$ is the selected variable to compare the results for each mesh tested.

Furthermore the boundary conditions that are applied on the fluid field are

$$
\left\{\begin{array}{c}
\left.\frac{\partial U_{u p}}{\partial z}\right|_{z=H_{\text {down }}+H_{u p}}=0 \\
\left.\frac{\partial U_{\text {down }}}{\partial z}\right|_{z=0}=0 \\
\left.\frac{\partial U_{u p}}{\partial z}\right|_{z=H_{\text {down }}}=\left.\frac{\partial U_{\text {down }}}{\partial z}\right|_{z=H_{\text {down }}}=\frac{\partial W}{\partial t}
\end{array}\right.
$$

which means that the particles that are in contact with the disk have the same velocity than the disk and that the particles that are in contact with the casing have velocity 0 , since the casing is considered to be totally rigid.

Extending the solution of the potential flow given in [3] for the lower fluid field, the term $p_{r_{0}}$ can be expressed as

$$
p_{r_{o}}=\rho_{F} r_{o} \sum_{n= \pm 1}^{\infty} \frac{A_{n}}{n} \times e^{j n \theta} \times e^{j \omega_{n} t}\left(\left(\omega_{n}+n \Omega_{\text {fluid-up }}\right)^{2} \operatorname{coth}\left(\frac{n H_{\text {up }}}{r_{0}}\right)+\left(\omega_{n}+n \Omega_{\text {fluid-down }}\right)^{2} \operatorname{coth}\left(\frac{n H_{\text {down }}}{r_{0}}\right)\right)
$$

Substituting Eqs. (8) and (5) into Eq. (4), the following equation is obtained to calculate the natural frequencies:

$$
\begin{aligned}
& \left(\left[\operatorname{coth}\left(\frac{n H_{u p}}{r_{o}}\right)+\operatorname{coth}\left(\frac{n H_{\text {down }}}{r_{o}}\right)\right] \frac{\rho_{F} r_{o}}{n}+\rho_{D} h_{D}\right) \omega_{n, f}^{2} \\
& \quad+\left(\left[\operatorname{coth}\left(\frac{n H_{u p}}{r_{o}}\right) 2 n \Omega_{\text {fluid-up }}+\operatorname{coth}\left(\frac{n H_{\text {down }}}{r_{o}}\right) 2 n \Omega_{\text {fluid-down }}\right] \frac{\rho_{f} r_{o}}{n}\right) \omega_{n \cdot f} \\
& \quad+\left(-\frac{D^{*}}{r_{0}} n^{4}+\left[\operatorname{coth}\left(\frac{n H_{\text {up }}}{r_{o}}\right) n^{2} \Omega_{\text {fluid-up }}^{2}+\operatorname{coth}\left(\frac{n H_{\text {down }}}{r_{o}}\right) n^{2} \Omega_{\text {fluid-down }}^{2}\right] \frac{\rho_{f} r_{o}}{n}\right)=0
\end{aligned}
$$

Only the positive solution of $\omega_{n f}$ is considered for n-positive and for n-negative. The following conclusions can be obtained analyzing Eqs. (9) and (5) in detail, regarding the natural frequencies and mode shapes of a rotating disk considering the surrounding fluid:

- When the disk is vibrating in a surrounding media with very low density compared to the density of the disk $\left(\rho_{f} \ll \rho_{D}\right)$, $p_{r_{0}} \approx 0$ and therefore Eq. (6) can be used to calculate the natural frequencies for each $n$.

- For the disk vibrating in air $\left(\rho_{f} \ll \rho_{D}\right)$, using Eq. (6) the solution of $\omega_{n, \text { air }}$ for $n$-positive and for n-negative is the same and therefore using Eq. (5) a standing wave is obtained vibrating at $\omega_{n, \text { air }}$ for each pair of $n$.

- In water, the density $\rho_{f}$ is much higher than in air and therefore Eq. (9) is considered. There is a different solution for $n$ positive and for n-negative. A physical explanation of the effect is that the rotating flow with respect to the disk has a different added mass effect in the wave with n-positive and in the wave with n-negative and therefore there are two natural frequencies for each diametrical mode in the stationary case: $\omega_{n \text {-positive,water }}<\omega_{n \text {-negative,water }}$.

- The viscosity of the fluid does not appear directly in Eq. (9). Nevertheless this parameter affects on $\Omega_{\text {fluid-up }}, \Omega_{\text {fluid-down }}$ and therefore affects on the values $\omega_{n \text {-positive,water, }} \omega_{n \text {-negative,water }}$

- Each solution of $\omega_{n, \text { water }}$ corresponds to a travelling wave on the disk. For n-positive, the wave rotates in the same direction than the disk and in the opposite direction for n-negative.

A similar effect is shown in [31], for a fluid-conveying pipe with periodic boundary conditions.

The influence of the radial gap in the axial vibration is not considered in Eq. (4). Nevertheless, in [22,23] is shown that for small radial gaps $\left(\left(r_{\text {casing }}-r_{\text {out }}\right) /\left(r_{\text {out }}\right)<0.05\right)$ the natural frequencies of the disk are decreased. To consider these cases, $r_{o}$ can be slightly increased with respect to the initial value calculated $\left(r_{o}=\sqrt{r_{i n} \times r_{\text {out }}}\right)$, minimizing the error between experimental and analytical values of the natural frequencies for the disk submerged, confined and non-rotating. 


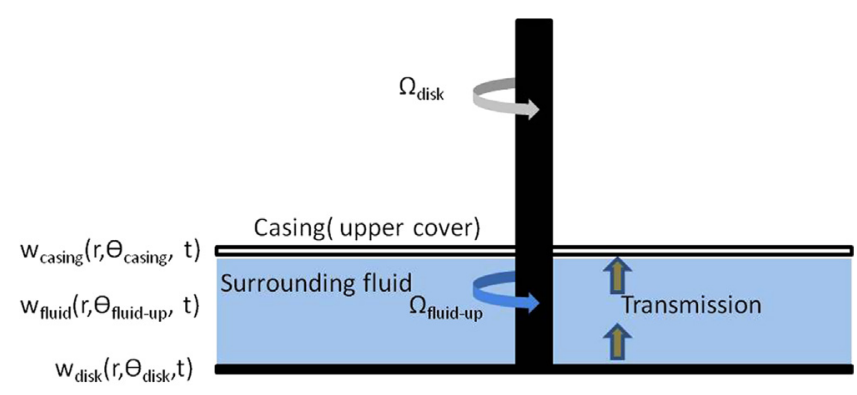

Fig. 2. Transmission from the rotating to the stationary frame.

\subsection{Transmission to the stationary frame}

In case of hydraulic turbomachinery, it is of paramount importance to know the dynamic response of the runner under operation, which means to know the natural frequencies and mode shapes of this structure when it is confined inside the casing and rotating. Due to the inaccessibility of this structure, it is very advantageous to measure its vibration from the stationary frame or casing.

In the previous section, the casing has been considered as a completely rigid structure to calculate $p_{r_{0}}$. Nevertheless, in the real cases, casings are not absolutely rigid. Therefore the following assumption for the velocity potential can be made for the particles in contact with the upper cover:

$$
\left.\left.\frac{\partial U_{u p}}{\partial z}\right|_{z=H_{\text {down }}+H_{u p}} \ll \frac{\partial U_{u p}}{\partial z}\right|_{z=H_{\text {down }}}
$$

If the displacement of the casing is significantly smaller than the displacement of the disk, the rigid condition of Eq. (8) can be used to calculate the added mass effect of the water and therefore the natural frequencies can be calculated according to Eq. (9). Furthermore, the condition expressed in Eq. (10) can be useful in order to measure the response of the disk with sensors placed on the casing.

The case of study has been presented in Fig. 1. To consider the transmission to the upper cover, Fig. 2 is considered.

The casing that covers the disk is not directly excited, but receives the vibration of the disk through the surrounding fluid. According to the general theory presented for fluid systems and structural vibrations in a fluid [28], if a structure vibrates with frequency $\omega$, the velocity potential of the surrounding fluid can be expressed, under the hypothesis for potential flow, as

$$
U=A \frac{c^{2}}{\omega} \eta(r, \theta) \sin \left(\omega t+\Psi_{f}\right)
$$

where $A$ is a dimensionless constant which specifies the amplitude of vibration, $c$ is the speed of sound, $\eta$ represents the mode shape of the fluid, and $\Psi$ is the phase angle. The dynamic variation of the pressure in the potential flow can be described as [30]

$$
p=-\rho_{F} \frac{\partial U}{\partial t}
$$

\subsubsection{Air}

The transmission to the casing depends on the dynamic pressure in the fluid and this depends on the fluid density according to Eq. (12). Therefore, when the surrounding fluid is air the transmission from the rotating structure to the casing is low and consequently it is difficult to extract information of the rotating frame with sensors that measure the vibration of the casing. In this case it is desirable to use non-contact sensors such as optical or proximity sensors that measure directly the vibration of the disk. Therefore, the following deduction is valid for a sensor placed on the stationary frame that measures directly the vibration of the disk.

Furthermore, in this case the diametrical modes of the disk are standing waves when they are viewed from the rotating system. Therefore, the vibration of the disk for a diametrical node $\mathrm{n}$ can be expressed in the rotating frame as

$$
w_{\text {disk }}\left(r, \theta_{\text {disk }}, t\right)=W_{n}(r) \cos \left(n \theta_{\text {disk }}\right) \cos \left(\omega_{n} t+\Psi_{\text {disk }}\right)
$$

For a non contact sensor that is measuring directly the vibration of the disk from the stationary frame (i.e. optical or proximity sensor), Eq. (13) can be rewritten using the relation between rotating and stationary frame (Eq. (1)):

$$
\begin{aligned}
w_{\text {disk }, \text { ref casing }}\left(r, \theta_{\text {casing }}, t\right)= & \frac{1}{2} W_{n}(r) \cos \left(\left(\omega_{n}+n \Omega_{\text {disk }}\right) t+n \theta_{\text {casing }}+\Psi_{\text {disk }}\right) \\
& +\frac{1}{2} W_{n}(r) \cos \left(\left(\omega_{n}-n \Omega_{\text {disk }}\right) t-n \theta_{\text {casing }}+\Psi_{\text {disk }}\right)
\end{aligned}
$$


According to Eq. (14), for a natural frequency in the rotating frame two natural frequencies are detected in the stationary frame:

$$
\left\{\begin{array}{l}
\omega_{n_{\text {stat,air_1 }}}=\omega_{n}-n \Omega_{\text {disk }} \\
\omega_{n_{\text {stat,air_2 }}}=\omega_{n}+n \Omega_{\text {disk }}
\end{array}\right.
$$

And the phase shift between two sensors (both measuring the same physical magnitude) in the stationary frame at the natural frequencies is:

$$
\Delta \alpha_{\omega_{n_{\text {stata,air }}}}=+n \Delta \theta_{\text {casing }} \text { and } \Delta \alpha_{\omega_{n_{\text {stata, air_-2 }}}}=-n \Delta \theta_{\text {casing }}
$$

where $\Delta \theta_{\text {casing }}$ is the spatial phase shift between two sensors in the peripheral direction of the casing and $\Delta \alpha$ is the phase difference between signals.

\subsubsection{Water}

For a high density fluid (water), the dynamic variation of pressure is not negligible (Eq. (12)) and therefore information of the disk vibration can be extracted when measuring the vibration of the casing. The viscosity does not appear directly in the deduced equations. Nevertheless this parameter has an influence on the damping and therefore on the resonance amplitude $W_{n}$, as shown in [23].

As mentioned before, in this case the types of mode shapes with no nodal circles that appear on the disk are travelling waves. Therefore for the disk vibration:

$$
w_{\text {disk }}\left(r, \theta_{\text {disk }}, t\right)=W_{n}(r) \cos \left(\omega_{n} t+n \theta_{\text {disk }}+\Psi_{\text {disk }}\right)
$$

The vibration of the particles of the fluid in contact with the disk can be expressed as

$$
w_{\text {fluid }}\left(r, \theta_{\text {fluid }}, t\right)=W_{n}(r) \cos \left(\omega_{n} t+n\left(\theta_{\text {fluid }}+\Omega_{\text {(disk)/(fluid })} t\right)+\Psi_{\text {fluid }}\right)
$$

here it is used that $\theta_{\text {disk }}=\left(\theta_{\text {fluid }}+\Omega_{\text {(disk)/(fluid })} t\right)$. If the displacement of the casing is much lower than the displacement of the disk the velocity potential of the flow can be described with the mode shape of the disk. Therefore,

$$
U=A \frac{c^{2}}{\omega} \sin \left(\omega_{n} t+n \Omega_{\text {(disk)/(fluid) }} t+n \theta_{\text {fluid }}+\Psi_{\text {fluid }}\right)
$$

Thus the dynamic pressure that the fluid exerts on the casing can be written as

$$
p=-\rho_{F} \frac{\partial U}{\partial t}=A_{p} \sin \left(\left(\omega_{n}+n \Omega_{\text {disk }}\right) t+n \theta_{\text {casing }}+\Psi_{\text {casing }}\right)
$$

where $A_{p}$ is the amplitude of the dynamic pressure and depends on the density of the fluid $\rho_{F}$. This pressure acts in the entire upper cover surface. If the frequency of the dynamic pressure (Eq. (20)) is well separated from a natural frequency of the casing, then the response of the casing should be dominated by the excitation shape of the dynamic pressure. In this case the transverse vibration of a point on the casing can be expressed as

$$
w_{\text {casing }}\left(r, \theta_{c}, t\right)=A_{\text {casing, } n}(r) \cos \left(\left(\omega_{n}+n \Omega_{\text {disk }}\right) t+n \theta_{\text {casing }}+\Psi_{\text {casing }}\right)
$$

where $A_{\text {casing, }, n}$ is the amplitude of vibration in the casing, which is much lower than the amplitude of vibration in the disk $\left(W_{n}(r)\right)$, since the casing has a forced vibration due to the disk vibration. The relation between a natural frequency viewed from the disk $\omega_{n}$ and viewed from the casing $\omega_{n, s t a t}$ is

$$
\omega_{n_{\text {stat,water }}}=\omega_{n, \text { disk }}+n \Omega_{\text {disk }}
$$

and the phase shift between two sensors on the casing installed at the same radius:

$$
\Delta \alpha_{\omega_{n_{\text {stat }, \text { water }}}}=n \Delta \theta_{\text {casing }}
$$

In Eqs. (22) and (23), $n$ can be positive or negative. The positive value of $n$ represents the lower natural frequency viewed from the rotating frame (Eq. (9)), which is a travelling wave rotating in the same direction than the disk. This frequency is translated to a higher frequency when viewed from the stationary frame as Eq. (22) shows. The negative value of $n$ represents the higher natural frequency viewed from the rotating frame, which is a travelling wave rotating in counter direction than the disk. This frequency is translated to a lower frequency when viewed from the stationary frame. This transmission characteristic is different than the transmission for the rotating disk in air, where each pair of n-positive and nnegative gives a standing wave viewed from the disk which is transmitted as two travelling waves in the stationary frame (see Eqs. (14)-(16)).

The associated mode shape observed in the casing (relative phase between sensors measuring the same magnitude) is also a travelling wave that moves in the same direction than the wave on the disk and with the same number of nodal diameters (Eq. (23)).

These deductions are also valid for a sensor that measures directly the disk vibration from the casing, such as an optical or non-contact sensor. If it is considered that there are no losses in the measurement process, the amplitude detected with 


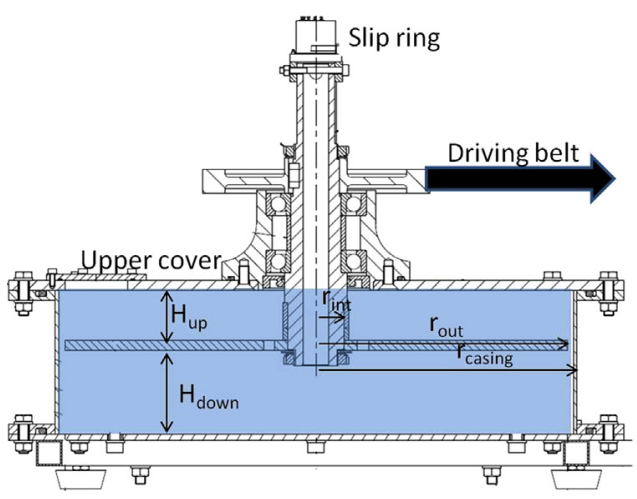

Fig. 3. Test rig without instrumentation.

this kind of sensors is the same than the amplitude of the disk vibration and therefore:

$$
A_{\text {casing, }, n}(r)=W_{n}(r)
$$

The deduced Eqs. (22) and (23) are valid so far the casing is not considered totally rigid and also when the casing has a negligible displacement compared to the displacement of the disk (flow affected only by the motion of the disk). Otherwise, the potential flow in the casing is affected by the deformation shape of the cover and the deformation shape of the disk. In this case, the equations deduced for the transmission are not anymore valid.

The displacement of the casing will be considerable if the fluid excites a frequency close to the natural frequency of the casing. Therefore, if it assumed that a natural frequency of the disk is well separated from a natural frequency of the casing, the transmission will be as described. Nevertheless, the dynamic response of casings in the real mechanical systems are usually very complex and with no peaky response, so the dynamic response of the casing itself has to be analyzed in detail in order to use the deduced equations.

\section{Experimental setup}

In order to verify the main conclusions of the analytical model, an experimental setup has been developed. It consists of a disk connected to a variable speed motor. When the disk is rotating, the excitation is performed from the rotating frame with a piezoelectric patch (PZT). The response is measured from the rotating frame with miniature accelerometers and from the stationary frame with different devices such as accelerometers, laser Doppler vibrometer or pressure sensors.

\subsection{Test rig}

\subsubsection{Disk}

The disk is made of stainless steel with an external radius $r_{\text {out }}=198 \mathrm{~mm}$ and a thickness of $h_{D}=8 \mathrm{~mm}$. The disk has a hole on its center in order to attach the shaft.

\subsubsection{Casing}

The casing is made of stainless steel. The inner diameter of the tank is $r_{\text {casing }}=205 \mathrm{~mm}$, which means that the radial gap from the disk to the casing is $7 \mathrm{~mm}$. The axial gap between the disk and the casing is $H_{\text {up }}=10 \mathrm{~mm}$ and $H_{\text {down }}=97 \mathrm{~mm}$. A scheme of the mounted disk in the casing without the instrumentation used is shown in Fig. 3.

In the upper cover of the casing several measurement devices can be installed in order to measure the response of the rotating disk from the stationary frame. An overview of the upper cover with some of the used sensors is shown in Fig. 4.

\subsubsection{Motor}

The motor is a Mavilor MLV-072, which is a variable speed motor. The rotating speed is controlled with a resolver (Mavilor Resolver 2T8). This parameter can be adjusted with a precision of $1 / 300 \mathrm{~Hz}$ using a control software given by the motor manufacturer. The vibrations of the motor are isolated from the rest of the test rig trough rubber mounting isolators installed between the motor support and the test rig.

When the disk is rotating in air the disk can rotate up to $10 \mathrm{~Hz}$. Nevertheless when the disk is rotating in water the velocity of the disk is limited to $8 \mathrm{~Hz}$ in order to avoid damages of the motor caused by the high power consumption.

\subsubsection{Slip ring}

Through the slip ring Michigan S10, the excitation and response signals are transmitted from the rotating to the stationary frame. This system is mounted at the tip of the shaft (Fig. 3). 


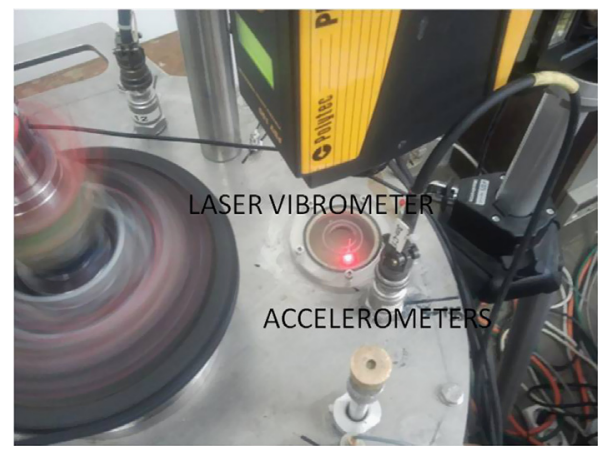

Fig. 4. Casing of the test rig with the mounted sensors.

\subsubsection{Data acquisition system}

A Bruel\&Kjaer Type 3038 module is used to acquire the signals. The maximum frequency acquisition is $25.6 \mathrm{kHz}$, much higher as needed for this study.

\subsection{Instrumentation}

\subsubsection{Accelerometers}

For the measurement of the response from the rotating frame miniature and submergible accelerometers Dytran 3006-A (sensitivity $100 \mathrm{mV} / \mathrm{g}$ ) have been used. They are directly screwed on the disk. The mass of the accelerometers is negligible small in comparison with the mass of the disk.

To measure the response from the stationary frame accelerometers Kistler $8752 \mathrm{~A} 50$ (sensitivity $100 \mathrm{mV} / \mathrm{g}$ ) have been glued on the upper cover.

\subsubsection{Piezoelctric patches (PZT's)}

For the excitation of the disk from the rotating frame one patch PI-876A12 $(61 \mathrm{~mm} \times 35 \mathrm{~mm})$ is glued on the disk with an epoxy component LOCTITE 454.

This patch works in a range of $-100 \mathrm{~V}$ to $250 \mathrm{~V}$. For the signal generation a NI-9263 module is used. This module can generate four independent analogical outputs with an amplitude of $-10 \mathrm{~V}$ to $10 \mathrm{~V}$. With an amplifier OEM-835 the analogical signal is amplified by 25 , so the send signal to the patch has the desired level. Also a signal for monitoring the excitation is send to the data acquisition system.

\subsubsection{Laser Doppler vibrometer}

To measure the response of the disk from the stationary frame, a laser Doppler vibrometer PDV-100 with adjustable sensitivity (sensitivity range of $200-8 \mathrm{~V} / \mathrm{ms}$ ). The laser is mounted on a tripod without any contact to the test rig in order to avoid influences of the casing vibration. In order to compare the magnitude of the laser with the magnitude of the accelerometers, the signal is differentiated. The focusing of the LDV is adjusted for the standing disk, comparing the amplitude of this sensor with an accelerometer placed on the disk (face to face). A glass window is made on the upper cover in order to use this sensor (Fig. 4).

\subsubsection{Pressure sensors}

Pressure sensors (KRISTAL Type 4295A2V34) are installed on the stationary frame measuring the dynamic pressure due to the disk vibration. They have to be powered with $18-36 \mathrm{~V}_{\mathrm{dc}}$ and they have a sensitivity of $5 \mathrm{~V} / \mathrm{bar}$ in a measurement range of $0-2$ bar.

Special holes have been screwed on the casing to install the pressure sensors.

\subsection{Position of the sensors}

\subsubsection{Rotating frame}

In the rotating frame one PZT and miniature accelerometers are placed. The nomenclature used for the accelerometers is AR- $X$, where $X$ is the angle related to the $0^{\circ}$ direction in counterclockwise direction, when the disk is attached to the shaft and viewing the test rig from the top. Four accelerometers (AR-0, AR-90, AR-180 and AR-210) have been used during the tests.

One patch is used to excite the disk from the rotating frame (P-0). The installed patch and accelerometers on the disk are shown in Fig. 5 (view from the bottom). 


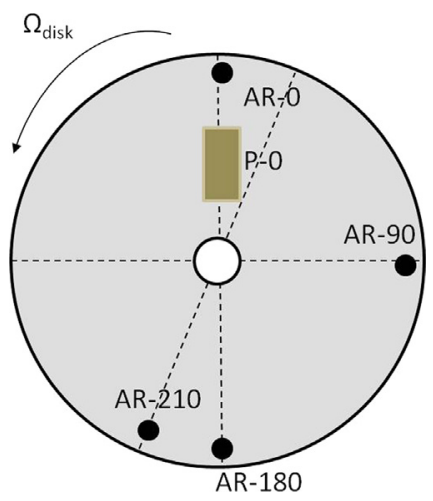

Fig. 5. Rotating disk with the position of accelerometers and piezoelectric patch (view from the bottom).

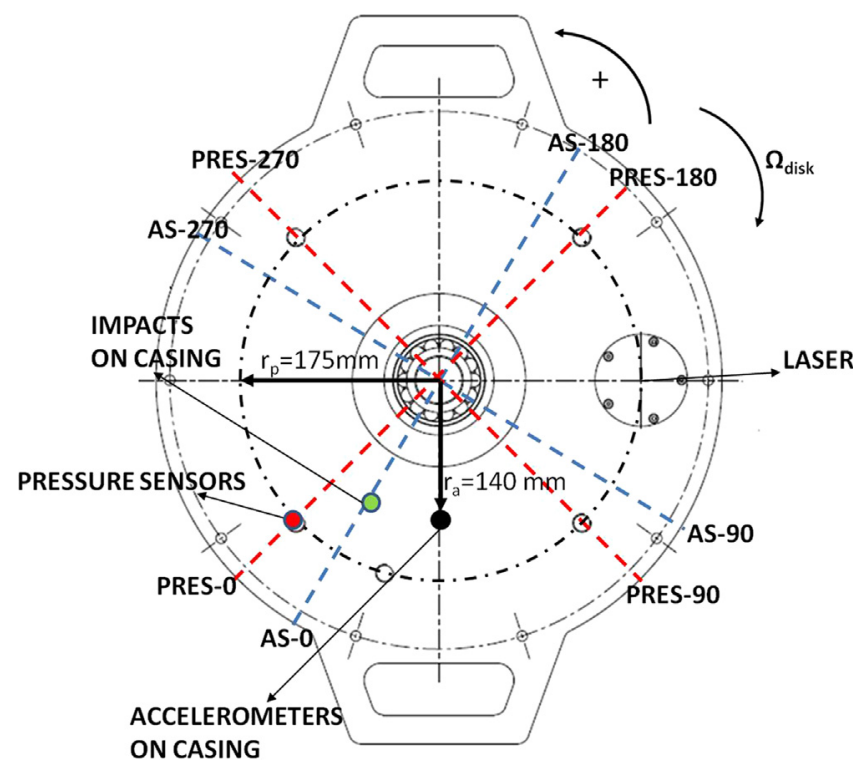

Fig. 6. Sensors on the stationary frame. View from the top.

\subsubsection{Stationary frame}

In the stationary frame (upper cover) pressure sensors, accelerometers and a laser Doppler vibrometer have been used. The accelerometer and pressure sensors are determined by its angular position with respect to its reference direction shown in Fig. 6. The radius for the accelerometers position is $140 \mathrm{~mm}$. Following accelerometers have been installed: AS-0, AS-45, AS-90, AS-135, AS-180, AS-210, AS-240.

In the same way, the position for pressure sensors is defined. The radius for the pressure sensors is $175 \mathrm{~mm}$. Three pressure sensors are installed: PRES-0, PRES-30, PRES-180.

The laser is installed in the shown position in Fig. 6. It is installed in the same radius than the pressure sensors.

In order to have a better view of the transmitted mode shape to the upper cover, different series of experimental tests have been performed leaving one accelerometer on the rotating frame (AR-0) and one accelerometer on the stationary frame (AS-0 in position S4 (Fig. 7)) as reference accelerometers. The disk is not stopped during the tests and its velocity is maintained constant in order to keep the boundary and environmental conditions invariant as possible.

The accelerometer on the rotating frame (AR-0) is used to check that the natural frequencies of the disk remain constant during all the tests (possible small changes in the boundary conditions such as temperature of water). The phase of the reference accelerometer on the stationary frame (AS-0) is used as reference phase for all the accelerometers on the stationary frame, when showing the transmitted mode shapes on the casing (Figs. 17-22).

Five series, changing the radial position of all other accelerometers placed on the casing, are performed. For each series, the disk is excited with a sweep excitation (with the PZT P-0) and the casing impacted with an instrumented hammer. The specified positions of the accelerometers and the impact positions are shown in Fig. 7.

As seen in Fig. 7, for each series an accelerometer every $22.5^{\circ}$ is located excepting on the line $135^{\circ}$ due to the interference of the Plexiglas window. The radial position of the accelerometers in the different series (S1-S5) is shown in Table 1 . 


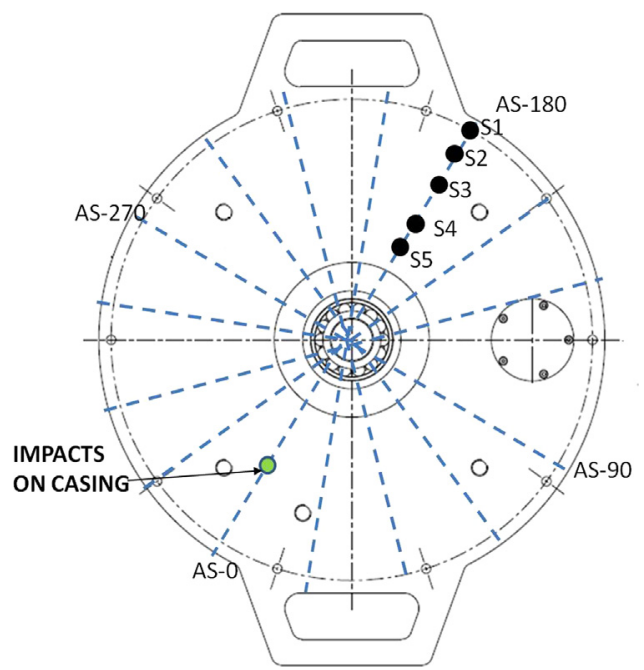

Fig. 7. Detailed study of the casing deformation shape. Position of the accelerometers.

Table 1

Radial position of the accelerometers in the different series (S1-S5).

\begin{tabular}{lllll}
\hline $\mathrm{S} 1$ & $\mathrm{~S} 2$ & $\mathrm{~S} 3$ & $\mathrm{~S} 4$ & $\mathrm{~S} 5$ \\
\hline $240 \mathrm{~mm}$ & $205 \mathrm{~mm}$ & $170 \mathrm{~mm}$ & $140 \mathrm{~mm}$ & $110 \mathrm{~mm}$ \\
\hline
\end{tabular}

\subsection{Tests performed}

\subsubsection{Sweep on the disk}

The transmission disk-casing is studied in detail for $\Omega_{\text {disk }}=0 \mathrm{~Hz}, 8 \mathrm{~Hz}$ and with air and water inside the tank. The modes that have been considered are the diametrical modes $n= \pm 2$ and $n= \pm 3$.

To excite the natural frequencies of the disk, the piezoelectric patch P-0 has been used. A slow sweep signal passing through the studied modes is used. Such a signal can be described as:

$$
y=A \sin \left(\left(\varsigma t+\omega_{0}\right) t\right) \text { for } 0<t<t_{\text {end }}
$$

When a patch works with this signal, it excites all the frequency bands from $\omega_{0}$ to $\varsigma t_{\text {end }}$.

This test is repeated for the position of the sensors shown in Fig. 6 and for all the series shown in Fig. 7.

\subsubsection{Impact on the casing}

To determine the response of the casing, it is impacted with an instrumented hammer. Five impacts have been performed for each test and the time signals have been averaged obtaining the response of the casing.

\section{Signal analysis}

\subsection{Peak hold method}

In Fig. 8, the procedure to analyze the excitation and response signals is shown for one sensor detecting a resonance of the disk. The time signal of the excitation P-0 (Fig. 8a) shows a slow sweep excitation. The time signal of AR-0 (Fig. 8a) shows that a resonance occur at certain time. To obtain the frequency content of these signals, a Hanning Window of $4 \mathrm{~s}$ (resolution $0.25 \mathrm{~Hz}$ ) is applied on the time signals. Since this window is shorter than the total length of the time signals, it is translated $0.2 \mathrm{~s}$ ( $5 \%$ of the window length) every average. In each average, the FFT is applied in both signals and superposed to the other averages with the maximum hold method, which considers only the maximum value for each frequency. In this way the frequency content of both signals is obtained (Fig. 8b). Using both response (AR-0) and excitation (P-0) signals, the frequency response is obtained (Fig. 8c). Natural frequencies are detected in precision looking at the peaks of the frequency response function (FRF). The corresponding mode shapes are obtained analyzing the relative phase of the accelerometers on the rotating frame and contrasting with the analytical model. In this way, natural frequencies and mode shapes are determined for the disk in the different situations tested.

This method is useful to determine the natural frequency and mode shapes of the disk when it is excited with a sweep excitation, nevertheless the time information is lost with this process. 

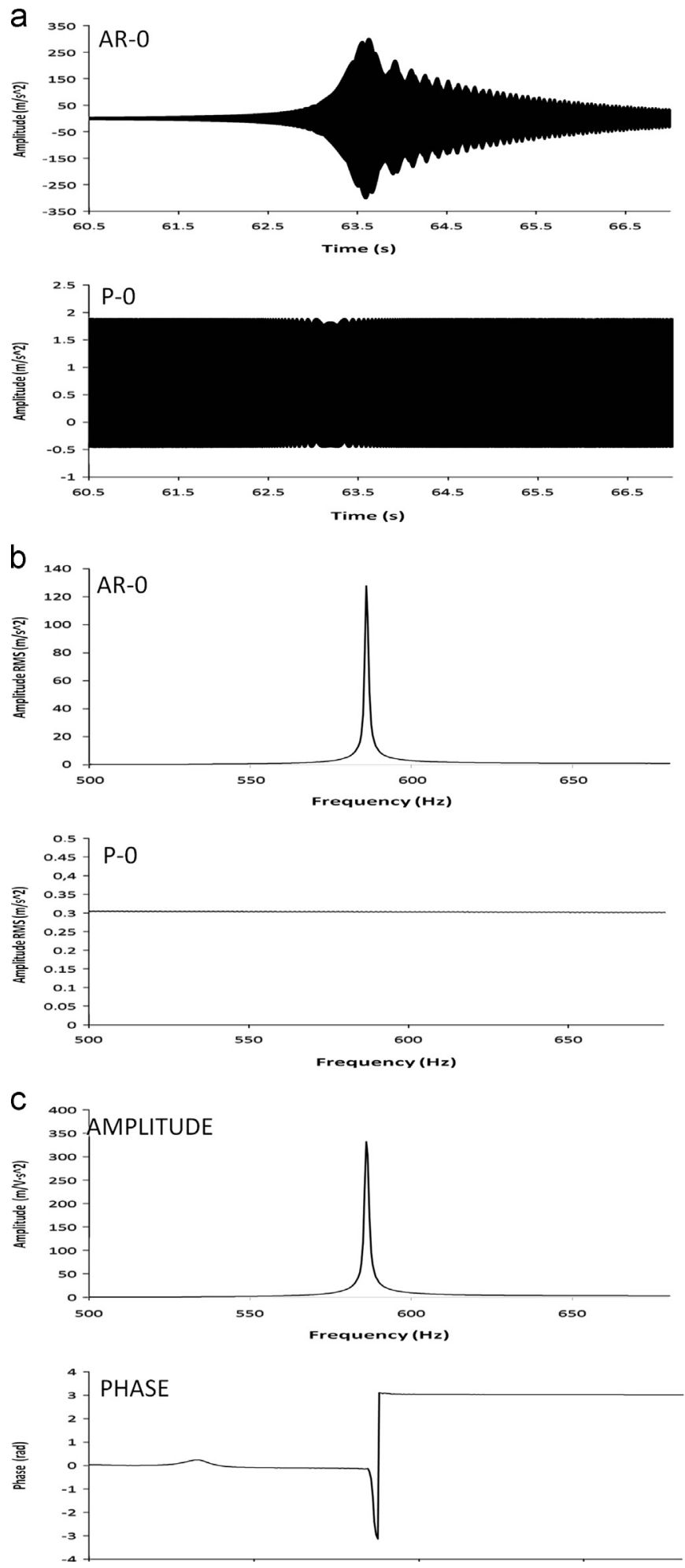

Fig. 8. Determination of the natural frequencies of the rotating disk. (a) Time signals. (b) Signals after FFT. (c) FRF amplitude and phase.

\subsection{Time-frequency using wavelets}

Wavelets have been used extensively in the last years to analyze temporary signals [32-35]. With respect to the STFT (short time Fourier transform), where it has to be chosen between good frequency resolution or time resolution [36], with 
a
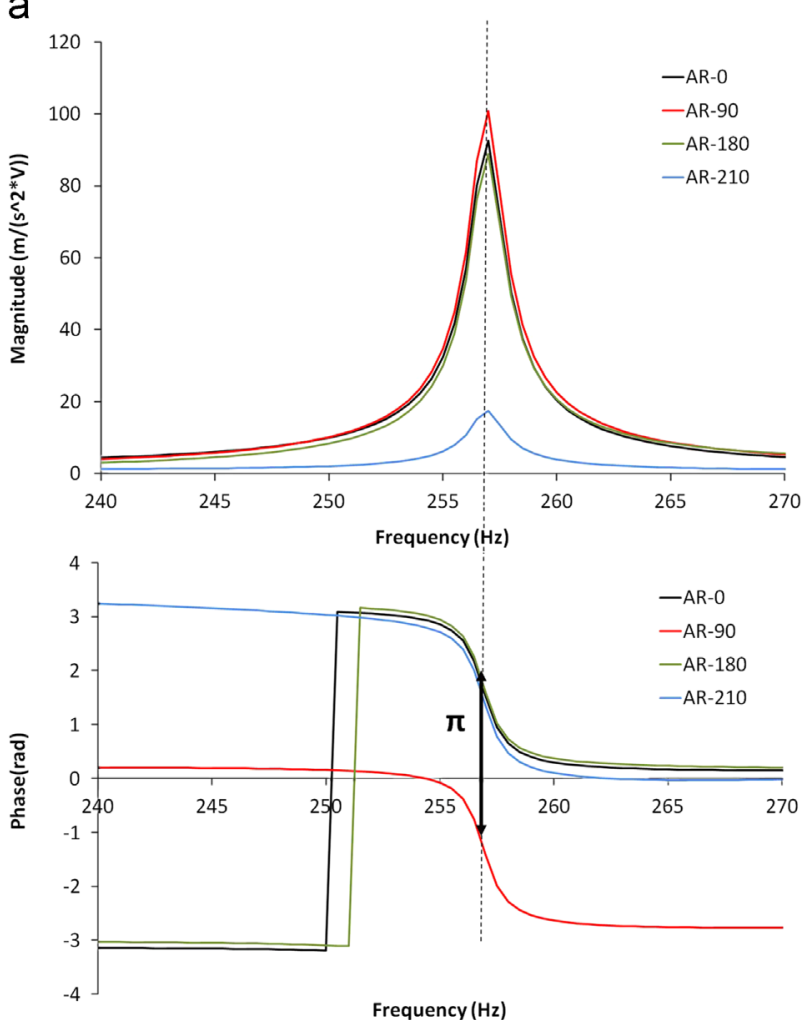

b
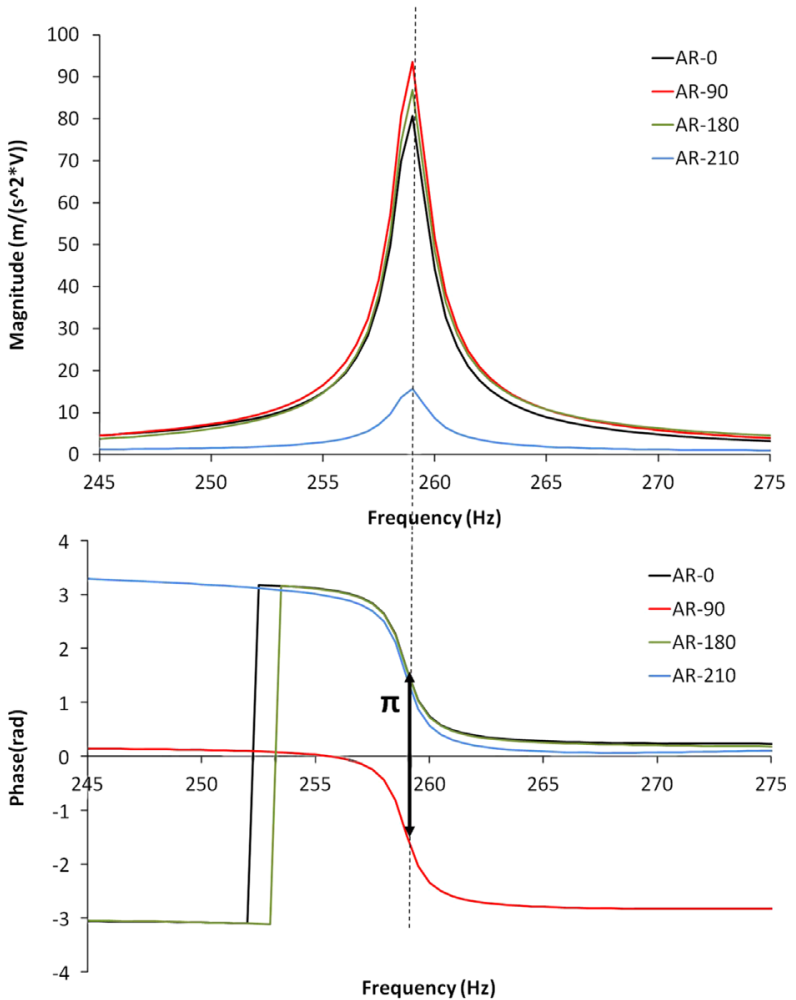

Fig. 9. Natural frequency $n= \pm 2$ in air from the rotating frame. (a) $\Omega_{\text {disk }}=0 \mathrm{~Hz}$, (b) $\Omega_{\text {disk }}=8 \mathrm{~Hz}$.

wavelets a multiresolution analysis, without losing important information of the signal [32], is performed. Good frequency resolution for the low frequency content of the signal and good time resolution for the high frequency content of the analysis is acquired. Wavelets have the general expression:

$$
\psi_{s, \tau}(t)=\frac{1}{\sqrt{s}} \psi\left(\frac{t-\tau}{s}\right)
$$

where $s$ scales the frequency of the wavelet and $\tau$ translates the wavelet in time. $\psi$ is the mother wavelet used. For this analysis the Morlet wavelet is chosen (see [32] for more details on this wavelet).

In the studied case, it is very useful to use time-frequency decomposition with wavelets in order to analyze the resonances that occur simultaneously in the rotating and in the stationary frame. This makes the transmission between both frames more comprehensible.

\section{Results}

\subsection{Natural frequencies and mode shapes from the rotating frame}

First, the natural frequencies and mode shapes of the modes $n= \pm 2$ and $n= \pm 3$ for the rotating disk in air and in water are determined. These are obtained for $\Omega_{\text {disk }}=0 \mathrm{~Hz}, 8 \mathrm{~Hz}$ from the rotating frame with the Peak hold method (Fig. 8), when the disk is excited with a sweep excitation using the piezoelectric patch.

The four accelerometers placed on the rotating frame are analyzed in amplitude and phase in order to determine the mode shapes. Furthermore, experimental results are compared with the analytical model.

\subsubsection{Air}

Applying the mentioned procedure the following amplitudes and phases are obtained for $\Omega_{\text {disk }}=0 \mathrm{~Hz}, 8 \mathrm{~Hz}$ when the disk is excited with a PZT (Fig. 9).

There is only a slight increase in the natural frequency comparing both cases. Analyzing the phase shift between sensors it can be seen that the mode is a mode $n= \pm 2$, which is a standing wave (all sensors in phase or in counterphase to each other). Extensively, the modes $n= \pm 3$ have been obtained. 

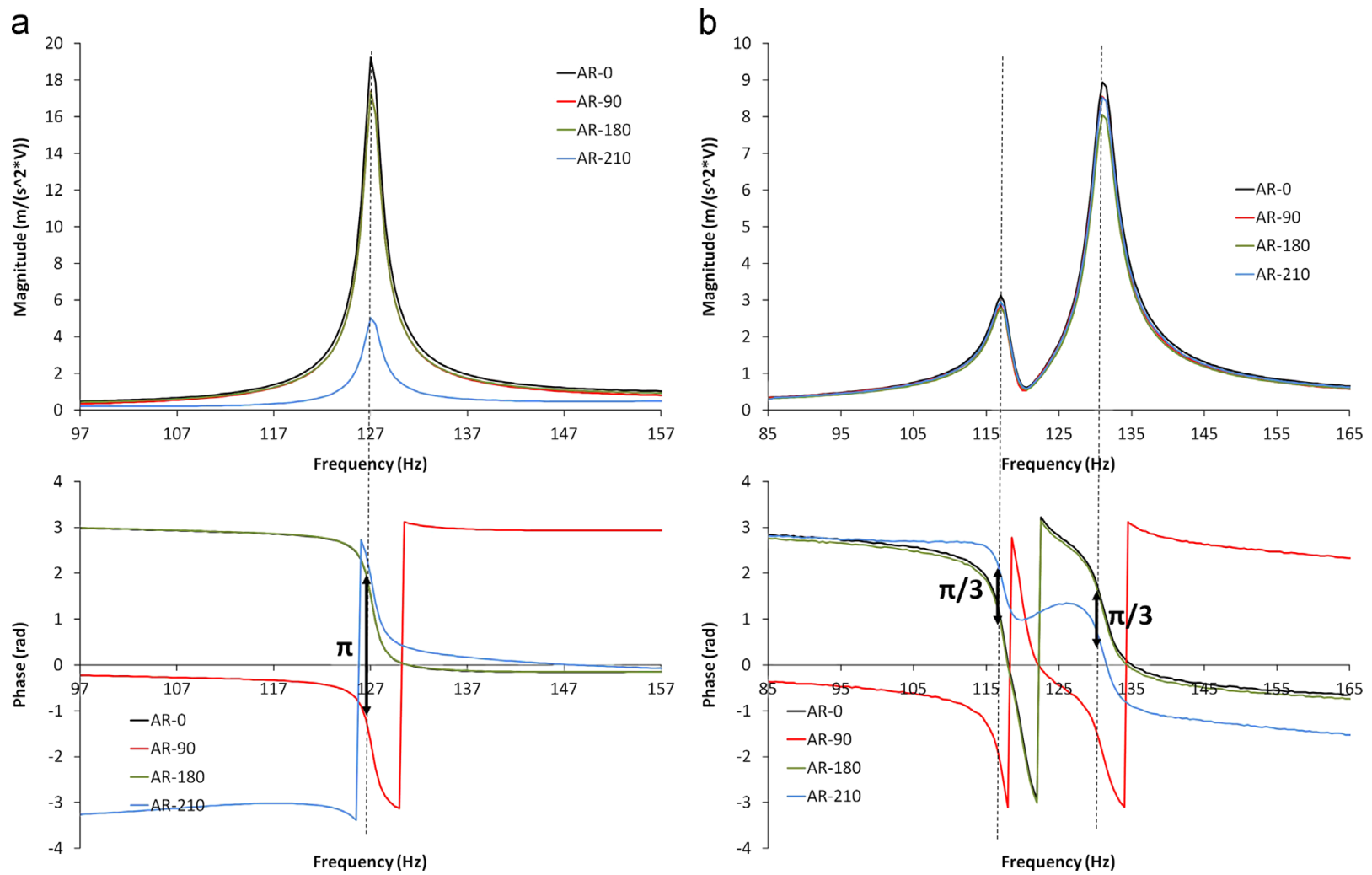

Fig. 10. Natural frequency $n= \pm 2$ in water from the rotating frame. (a) $\Omega_{\text {disk }}=0 \mathrm{~Hz}$, (b) $\Omega_{\text {disk }}=8 \mathrm{~Hz}$.

Table 2

Numerical values for the natural frequencies in $\mathrm{Hz}$ for the modes: (a) $n= \pm 2$, (b) $n= \pm 3$.

\begin{tabular}{|c|c|c|c|c|}
\hline \multirow[t]{2}{*}{ (a) } & \multicolumn{2}{|c|}{$\Omega_{\text {disk }}=0 \mathrm{~Hz}$} & \multicolumn{2}{|c|}{$\Omega_{\text {disk }}=8 \mathrm{~Hz}$} \\
\hline & $n=2$ & $n=-2$ & $n=2$ & $n=-2$ \\
\hline Air experimental & 257.43 & & 258.72 & \\
\hline Air analytical & 260.03 & & 260.03 & \\
\hline Error (\%) & -1.0 & & -0.5 & \\
\hline Water experimental & 127.05 & & 117.41 & 132.26 \\
\hline Water analytical & 122.18 & & 114.78 & 129.33 \\
\hline Error $(\%)$ & 4.0 & & 2.3 & 2.3 \\
\hline \multirow[t]{2}{*}{ (b) } & \multicolumn{2}{|c|}{$\Omega_{\text {disk }}=0 \mathrm{~Hz}$} & \multicolumn{2}{|c|}{$\Omega_{\text {disk }}=8 \mathrm{~Hz}$} \\
\hline & $n=3$ & $n=-3$ & $n=3$ & $n=-3$ \\
\hline Air experimental & 590.30 & & 592.12 & \\
\hline Air analytical & 585.07 & & 585.07 & \\
\hline Error (\%) & 0.9 & & 1.2 & \\
\hline Water experimental & 321.16 & & 309.11 & 329.96 \\
\hline Water analytical & 345.82 & & 337.04 & 354.35 \\
\hline Error $(\%)$ & -7.1 & & -8.2 & -6.9 \\
\hline
\end{tabular}

\subsubsection{Water}

The same procedure is applied when the disk is submerged in water and rotating (Fig. 10).

Now an important change is observed when comparing the standing and the rotating case. In the standing case there is only one peak, which is a standing wave $n= \pm 2$. In the rotating case, two peaks appear which corresponds to a travelling wave as seen in the phase difference of sensor AR-210. This sensor is placed at $30^{\circ}$ from the sensor AR-180 and therefore 
a

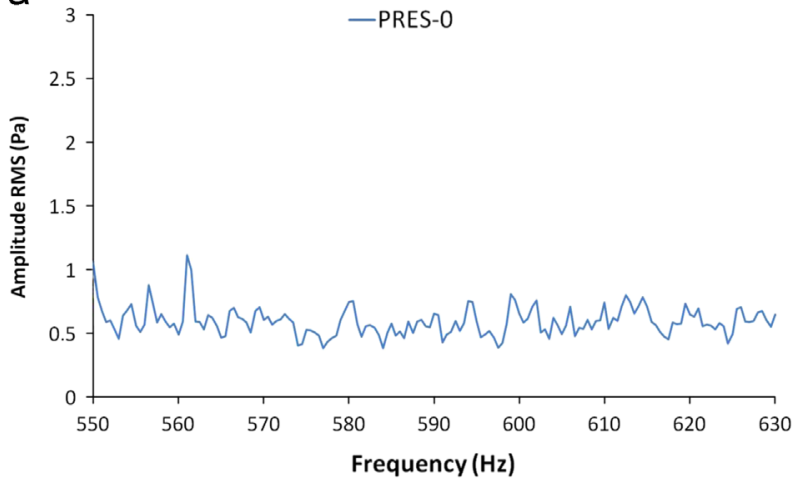

b

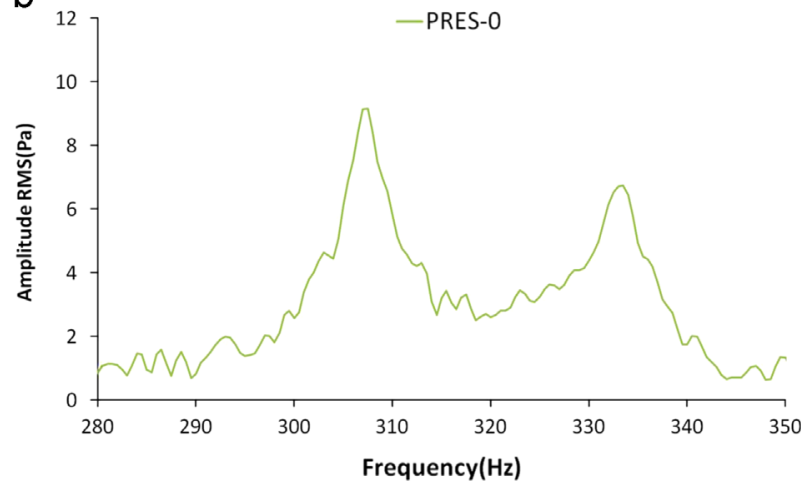

Fig. 11. Amplitude of the pressure detected with PRES-0 for the mode $n= \pm 3$ : (a) air and (b) water.

a

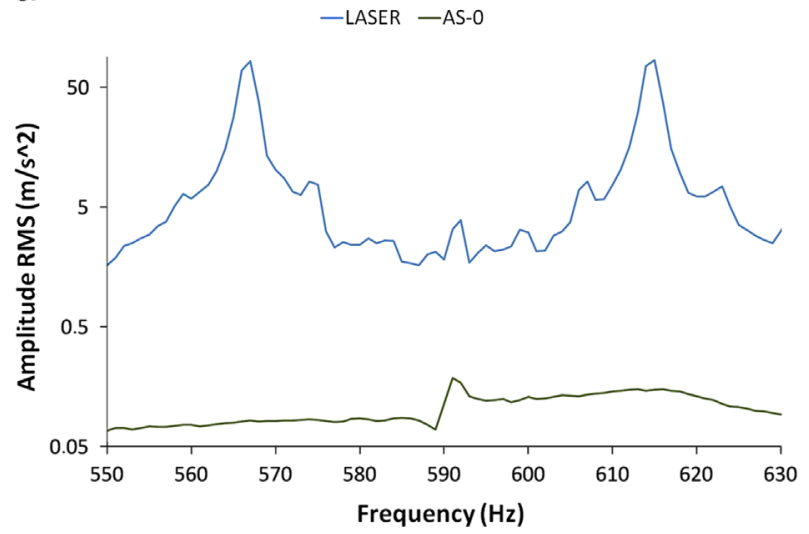

b

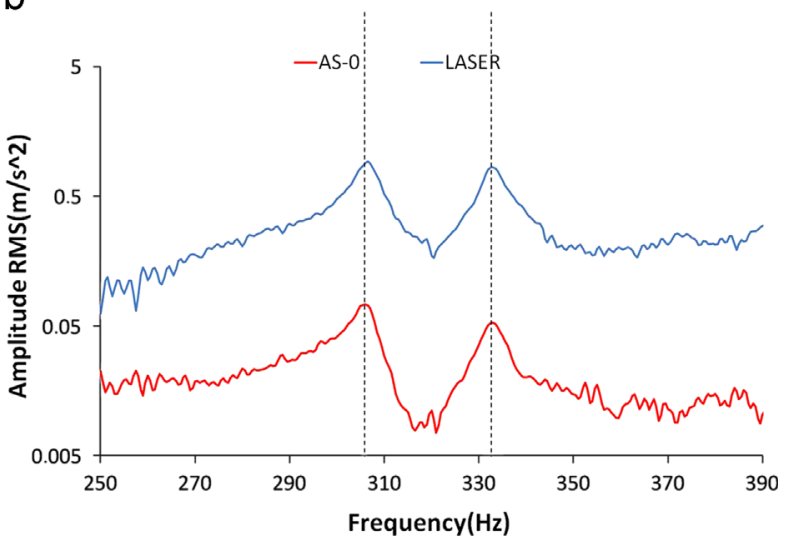

Fig. 12. Amplitude of vibration detected with LASER and AS-0 for the mode $n= \pm 3$ : (a) air and (b) water.

according to Eq. (23) the phase shift between them for a mode $n= \pm 2$ should be $60^{\circ}(\pi / 3)$ for one peak and $-60^{\circ}$ for the other peak, as shown in Fig. 10.

A comparison between analytical and experimental results for the natural frequencies in air and in water for $0 \mathrm{~Hz}$ and $8 \mathrm{~Hz}$ is shown in Table 2 .

\subsection{Detection of the disk natural frequencies and mode shapes from the stationary frame with the peak hold method}

The study of the transmission will be shown for the rotating disk $\left(\Omega_{\text {disk }}=8 \mathrm{~Hz}\right)$ in air and in water for the mode $n= \pm 3$. For other rotating speeds and diametrical modes the conclusions are equivalent so far the assumptions of the analytical model presented are accomplished.

The transmission mechanism is shown in Fig. 2. In order to detect the modal parameters of the rotating disk with sensors that measure the vibration of the upper cover, the fluid has to excite the upper cover with enough amplitude and this part cannot be totally rigid (Eq. (10)). According to Eq. (12), the dynamic pressure that the fluid exerts on the upper cover depends on the density of the fluid. Therefore, for the case that the fluid is air this pressure should be much lower than for the case that the fluid is water. This is shown in Fig. 11 (response of the pressure sensors).

As shown in Fig. 11, when the disk rotates in air nothing is seen in the frequency band $\omega_{n} \pm n \Omega_{\text {disk }}$. Nevertheless in water, two clear peaks are seen which corresponds to the peaks detected from the stationary frame $\omega_{-3, \text { disk }}-3 \Omega_{\text {disk }}$ and $\omega_{+3}$, disk $+3 \Omega_{\text {disk. }}$. This confirms that in the water case, since the density is much higher than in air, the fluid transmits the vibration of the disk from the rotating to the stationary case.

The comparison of the laser sensor and accelerometer AS-0 in air and in water is shown in Fig. 12.

When the disk is rotating in air (Fig. 12a), it is much easier to detect the natural frequencies from the stationary frame with optical or proximity sensors that measure the vibration of the disk directly, because the fluid does not excite the upper cover (Fig. 11). For the accelerometer AS-0 a small peak is detected, which coincides with the peak detected in the rotating frame. The explanation of this peak is the axial transmission of the mode $n= \pm 3$ through the mechanical system, i.e. the vibration of the disk is transmitted to the casing through the bearings on the shaft (see Fig. 3). Nevertheless, the amplitude detected with the accelerometer AS-0 is approximately 1/1000 of the amplitude 
a

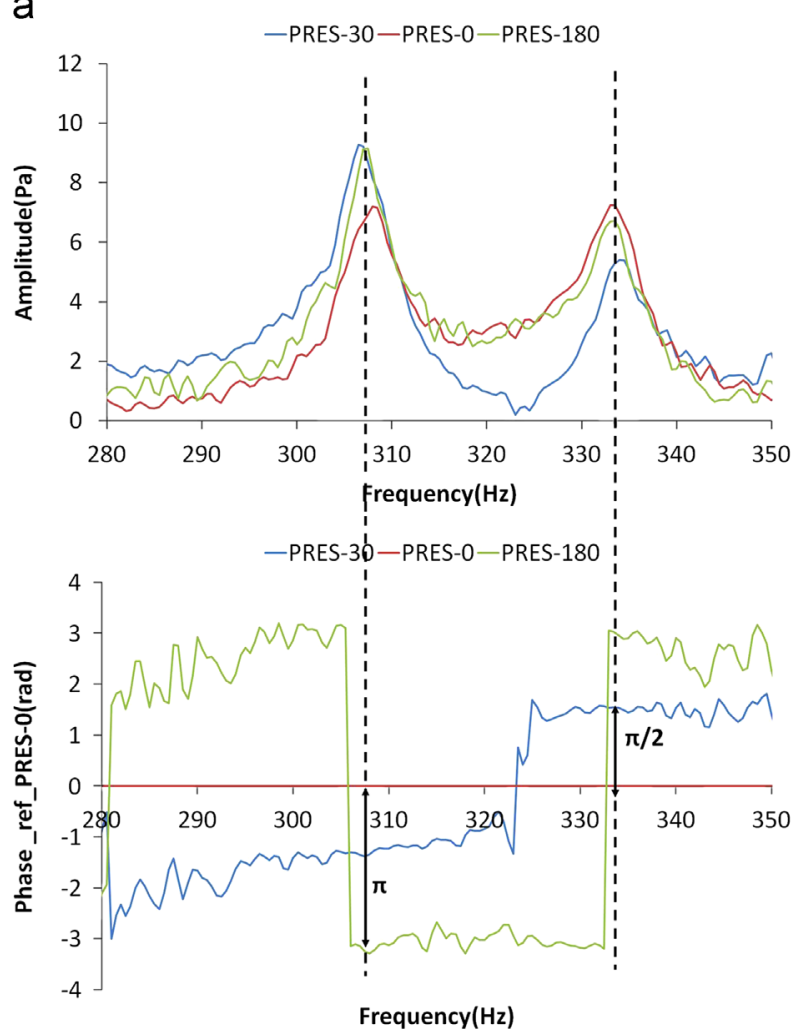

b

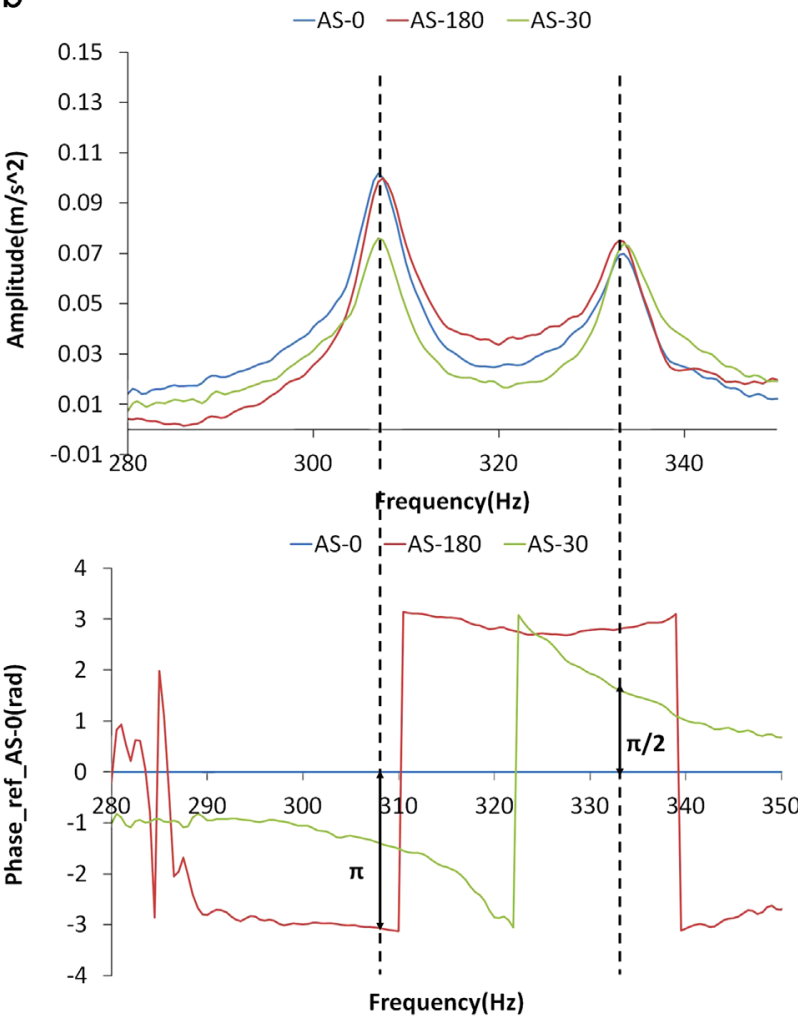

Fig. 13. (a) Amplitude of the pressure sensors and phase with respect to pressure sensor "PRES-0". (b) Amplitude of the accelerometers and phase with respect to accelerometer "AS-0".

a

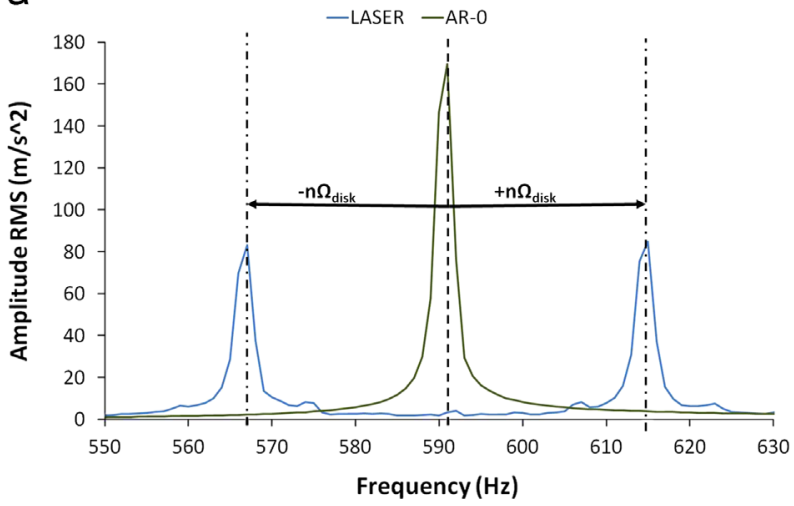

b

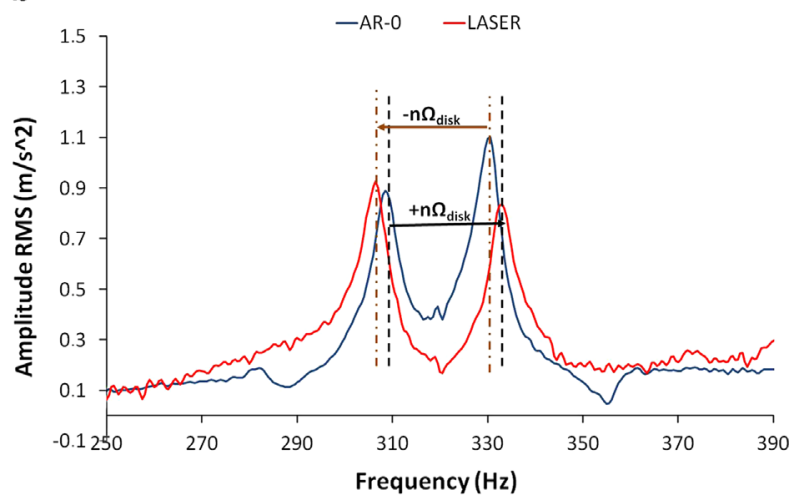

Fig. 14. AR-0 and LASER for the modes $n= \pm 3$ : (a) air, (b) water.

detected from the rotating frame, which means that the transmission through a stiff system as the shaft bearings and casing is very low.

For the case that the disk vibrates in water (Fig. 12b), two clear peaks are detected with the laser and with the accelerometer AS-0. These peaks are the same as those ones detected with the pressure sensor (Fig. 11b). In this case, the disk transmits the vibration to the casing though the water between them. Comparing amplitudes of the laser and amplitudes of the accelerometer it can be seen that the amplitude of the laser is much higher, as the laser is pointing directly on the disk and the accelerometer is measuring the vibration through the casing (see Eq. (24)).

To determine the mode shapes of the disk with sensors on the upper cover, the phase difference (Eq. (23)) between sensors has to be measured. This will be performed only for the water case, since in air the natural frequencies are not transmitted. Three pressure sensors and three accelerometers from those ones installed on the upper cover are used to analyze the mode shape (Fig. 13). 
a

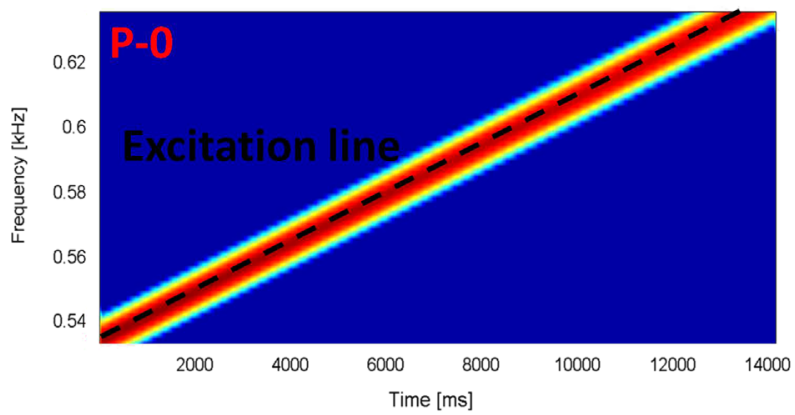

SCALO, Morlet wavelet, NhO=170.2939, N=64, log. scale, pcolor, Thld $=5 \%$

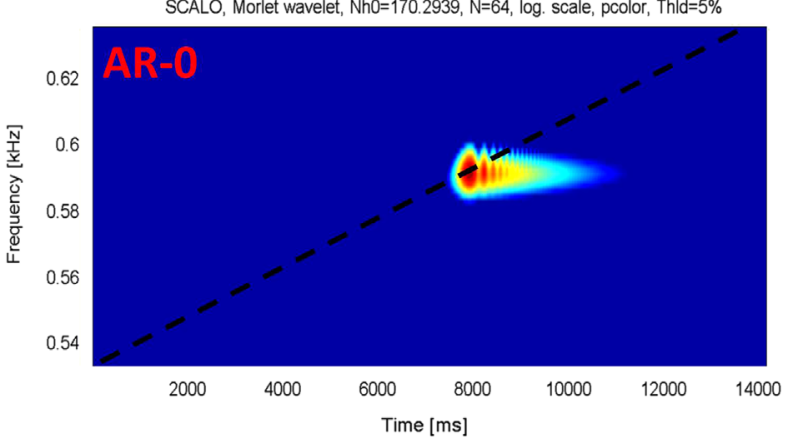

SCALO, Morlet wavelet, $\mathrm{NhO}=170.2939, \mathrm{~N}=64$, log. scale, pcolor, Thld $=5 \%$

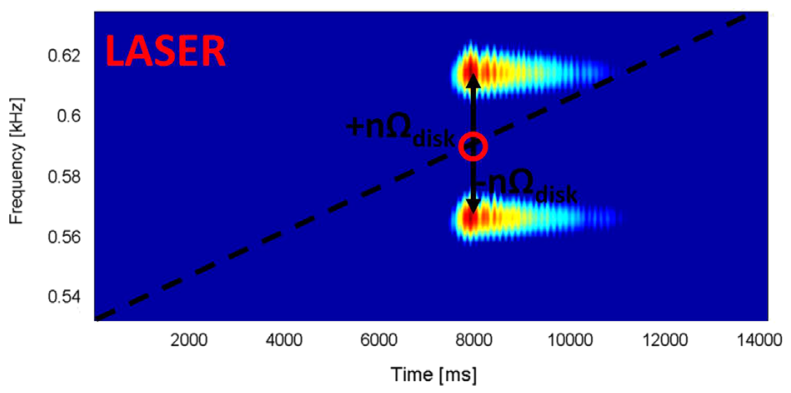

b

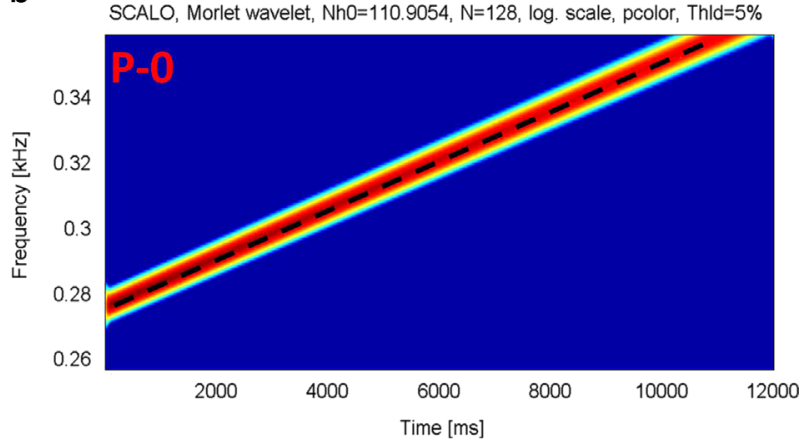

SCALO, Morlet wavelet, $\mathrm{NhO}=110.9054, \mathrm{~N}=128, \log$, scale, pcolor, Thld $=5 \%$

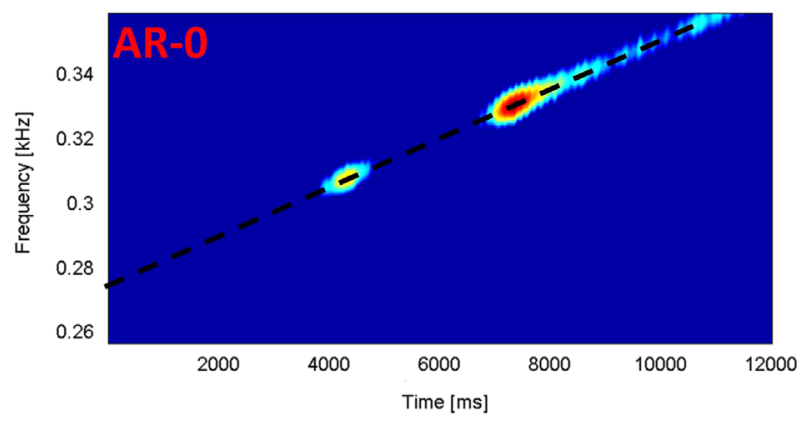

SCALO, Morlet wavelet, $\mathrm{NhO}=110.9054, \mathrm{~N}=128, \log$. scale, pcolor, Thld $=5 \%$

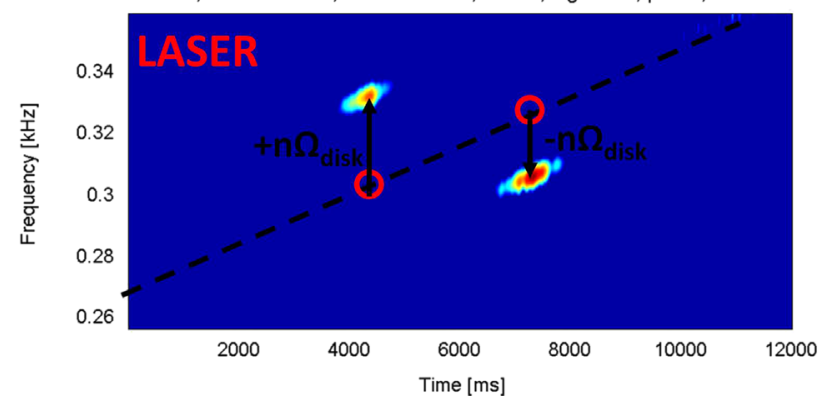

Fig. 15. Time-frequency plot of P-0, AR-0 and LASER for the mode $n= \pm 3$ : (a) air, (b) water.

As predicted by Eq. (23), the phase shift between the pressure sensors correspond to the modes $n= \pm 3$. In both peaks the phase of two sensors at $180^{\circ}$ (PRES-0 and PRES-180) is $\pi$ rad. With these two sensors, the direction of the travelling wave cannot be determined. Considering two sensors at $30^{\circ}$ from each other (PRES-0 and PRES-30) the phase shift should be $\pm 90^{\circ}$ or $\pm \pi / 2$ rad. In the first peak a phase shift of $-\pi / 2$ is observed, which means that the wave is travelling in direction from PRES-0 to PRES-30. This correspond to a travelling wave rotating in the opposite direction than the disk, corresponding to the mode $n=-3$ in the rotating frame. In the second peak a phase shift of $\pi / 2$ is observed, which indicates the mode $n=3$ in the rotating frame, according to the rotating direction of the disk.

The same information can be extracted when analyzing the accelerometers in the same relative position to each other (Fig. 13b).

The relation between the peaks detected from the rotating frame and the peaks detected from the stationary frame can be appreciated in Fig. 14, where the peaks of LASER and AR-0 are shown for the disk rotating in air and in water with $\Omega_{\text {disk }}=8 \mathrm{~Hz}$.

When the disk rotates in air, two clear peaks are detected with the laser separated at $\pm n \Omega_{\text {disk }}$ from the natural frequency detected from the rotating frame (green curve in Fig. 14a). As seen in this figure the amplitude of the laser vibration in the two peaks is approximately the half of the amplitude measured with the accelerometer AR-0 as predicted by Eq. (14).

When the disk rotates in water, since the laser is pointing directly on the disk, the amplitude of the resonance detected by this sensor is approximately the same amplitude as detected with the accelerometer on the rotating frame (Eq. (24)).

The frequency shift between rotating and stationary frame ( $\pm n \Omega_{\text {disk }}$ ) can be seen in Fig. 14 for the disk rotating in air and for the disk rotating in water. Nevertheless, this information can be extracted from this plot, because sensors measuring in 
a

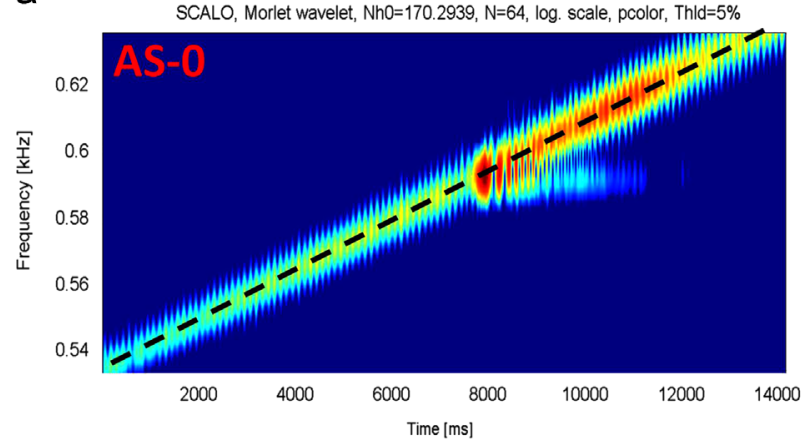

SCALO, Morlet wavelet, Nh0=170.2939, N=64, log. scale, pcolor, Thld $=5 \%$

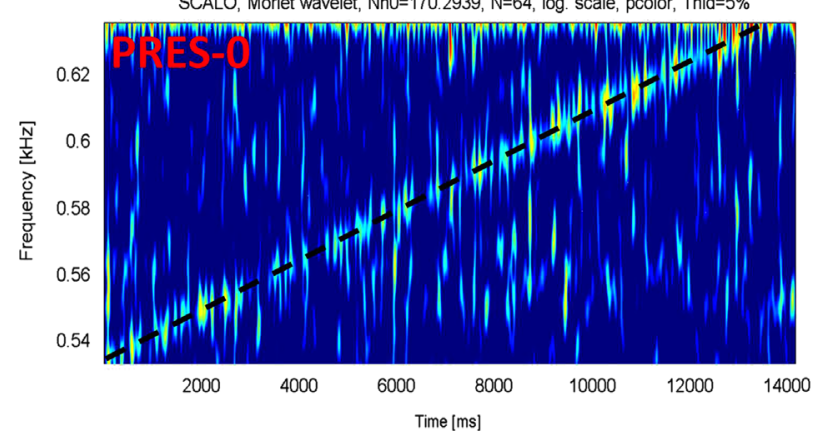

b

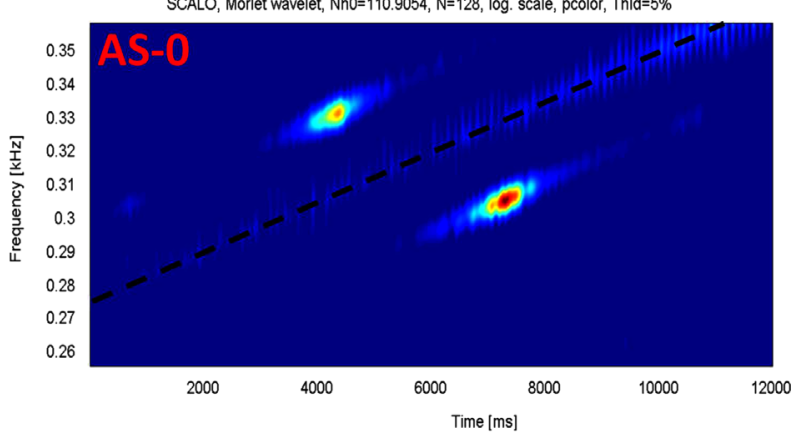

SCALO, Morlet wavelet, NhO=110.9054, $\mathrm{N}=128$, log. scale, pcolor, Thld $=5 \%$

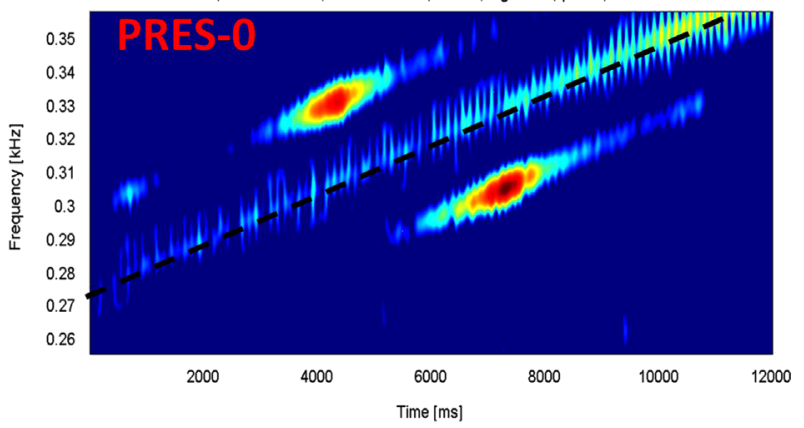

Fig. 16. Time-frequency plot of AS-0 and PRES-0 for the mode $n= \pm 3$ : (a) air, (b) water.

the stationary and rotating frame are plotted together. With this kind of representation, the temporal information is not explicitly seen and therefore it is difficult to understand the transmission from the rotating to the stationary frame if only sensors on the stationary frame are used.

For this reason, in order to study the transmission, it is better to analyze the resonances with a time frequency plot.

5.3. Detection of the disk natural frequencies and mode shapes from the stationary frame with time-frequency analysis using wavelets

To analyze the different kind of transmission that occurs for the rotating disk in air and for the rotating disk in water the excitation (P-0), the response of the rotating system (AR-0) and the detection from this response from the rotating frame (LASER) is shown in a time-frequency plot. The frequency content of the signals has been obtained with the use of wavelets [37] (Fig. 15). As shown in this figure, the electrical signal send to the patch is constant for the frequency band excited in both cases. In air (Fig. 15a), the frequency band excited is $540-640 \mathrm{~Hz}$ while in water (Fig. 15b) this frequency band is 280$350 \mathrm{~Hz}$.

In air, approximately at $590 \mathrm{~Hz}$ the excitation passes through the mode $n= \pm 3$ and the resonance is clearly detected with the accelerometer AR-0 (Fig. 15a) placed on the rotating frame. At the same time, two resonances occur on the stationary frame and they are clearly detected with the laser. They are separated $\pm n \Omega_{\text {disk }}$ from the resonance on the rotating frame, as predicted by Eq. (15).

Considering the rotating disk in water (Fig. 15b), with the accelerometer on the rotating frame (AR-0), it is clearly seen that two resonances occur in the rotating frame for the mode $n= \pm 3$. Since in this case, the mode shape is a travelling wave as shown in the analytical model, each of these two resonances are transmitted to one resonance on the stationary frame. The lower frequency (n-positive) in the rotating frame is transmitted to a higher frequency in the stationary frame and the higher frequency (n-negative) in the rotating frame is transmitted to a lower natural frequency in the stationary frame. The resonances on the stationary frame are clearly detected with the Laser. This plot confirms the behavior predicted by Eq. (22).

For accelerometers and pressure sensors on the upper cover, the frequency shift measured is the same as the frequency shift measured with the laser for the rotating disk in water (Fig. 16b); i.e. the two resonances detected on the stationary frame occur at different time. In these plots, it is shown that the detection is not as clear as with the laser, as appreciated with the Peak-hold analysis.

In air, the resonance is slightly detected with the accelerometer AS- 0 and without modulation as commented in the Peak-hold analysis (Fig. 12). With the pressure sensor PRES-0 nothing clear is seen, since in this case the density of the fluid is very low. 
Table 3

Type and number of waves for each diametrical mode of the disk depending on the sensor position.

\begin{tabular}{lll}
\hline & Rotating frame & Stationary frame \\
\hline Air & One standing & Two travelling \\
Water & Two travelling & Two travelling \\
\hline
\end{tabular}

a

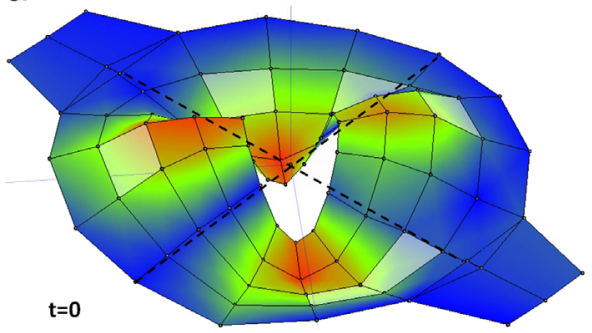

b

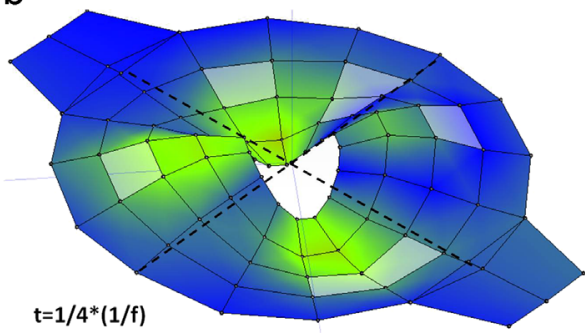

Fig. 17. Mode $n= \pm 2$ detected from the casing: $\Omega_{\text {disk }}=0 \mathrm{~Hz}$.

a

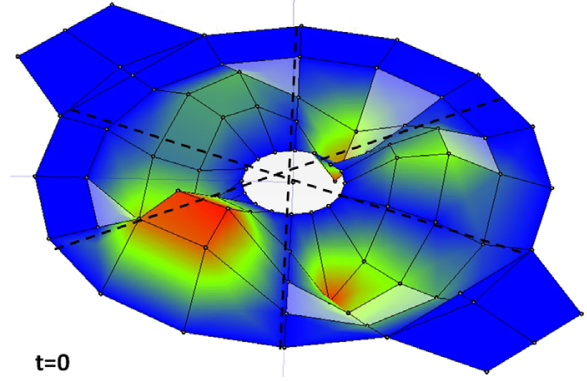

b

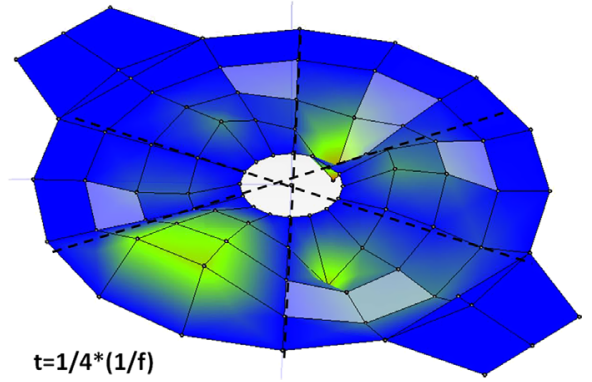

Fig. 18. Mode $n= \pm 3$ detected from the casing: $\Omega_{\text {disk }}=0 \mathrm{~Hz}$.

It is shown that the transmission from the rotating to the stationary frame is much easier to understand with a timefrequency plot, where the correspondence between resonances that occur at the same time is seen.

The main conclusion is that, in water for each diametrical mode of the disk two resonances occur in the rotating frame at different time and these are transmitted each to one peak in the stationary frame, since these are travelling waves. In air the resonance which is a standing wave is transmitted simultaneously to two travelling waves in the stationary frame. To summarize the type of waves depending on the detection frame, Table 3 is shown.

\subsection{ODS (operating deflection shape) of the upper cover for the resonance peaks (rotating disk in water)}

To have a better view of the mode shape transmitted to the upper cover, the experimentation shown in Fig. 7 is performed. The following ODS are obtained looking at the motion of all the used accelerometers simultaneously, vibrating at the resonance peaks detected in the stationary frame. The holes used for the pressure sensors and for the Laser are schematized in the geometry of the upper cover.

In the non rotating disk case $\left(\Omega_{\text {disk }}=0 \mathrm{~Hz}\right.$ ), the modes $n= \pm 2 ; \pm 3$ of the disk (stationary waves) can be clearly seen.

As seen in both cases, the nodal diameters do not rotate in time, and this indicates the presence of standing mode shapes on the disk with all the points moving in phase or in counterphase to each other.

When the disk is rotating, two travelling waves are detected from the stationary frame for each diametrical node $n$. The lower frequency of the rotating frame, which corresponds to the higher frequency in the stationary frame, rotates in the same direction than the disk. The higher frequency of the rotating frame, which corresponds to the lower frequency in the stationary frame, rotates in the opposite direction than the disk.

As seen in Figs. 19 and 20 the nodal diameters rotate in counter direction than the disk. In 1/4 of the period they have rotated approximately $45^{\circ}$. The mode $n=-2$ (Fig. 19) has rotated in the opposite direction than the disk and the mode $n=2$ (Fig. 20) in the same direction.

Figs. 21 and 22 show the modes $n=3$ and $n=-3$. In these two figures, the ODS represented correspond to the peaks shown in Fig. 14. As seen in Fig. 21 the nodal diameters rotate in counter direction than the disk. In $1 / 4$ of the period they have rotated approximately $30^{\circ}$. Fig. 22 shows the travelling wave rotating in the opposite direction (mode $n=3$ ). 

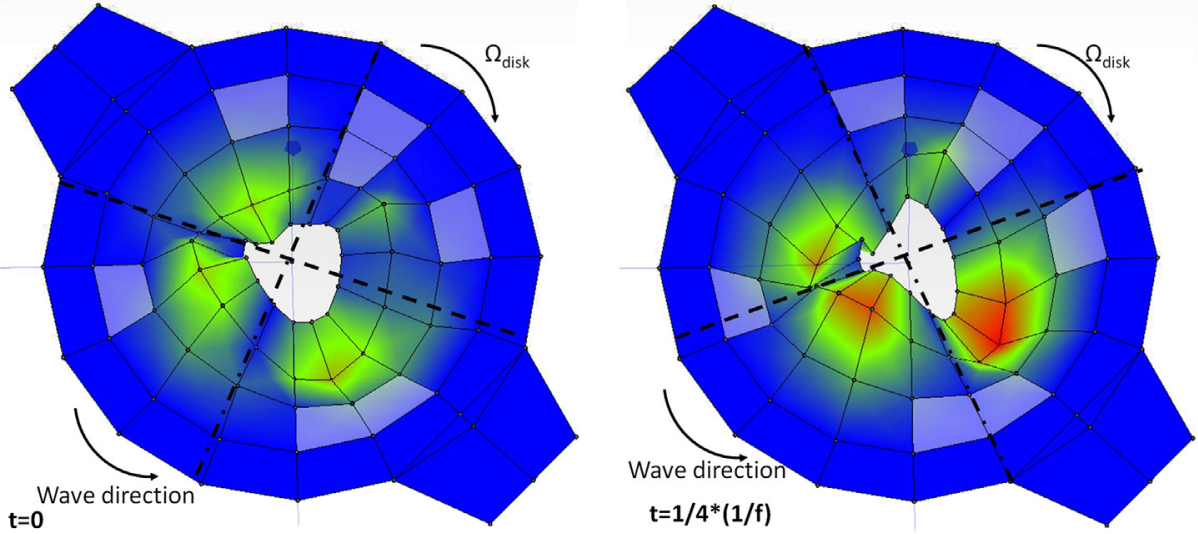

Fig. 19. Mode $n=-2$ detected from the casing: $\Omega_{\text {disk }}=8 \mathrm{~Hz}$.
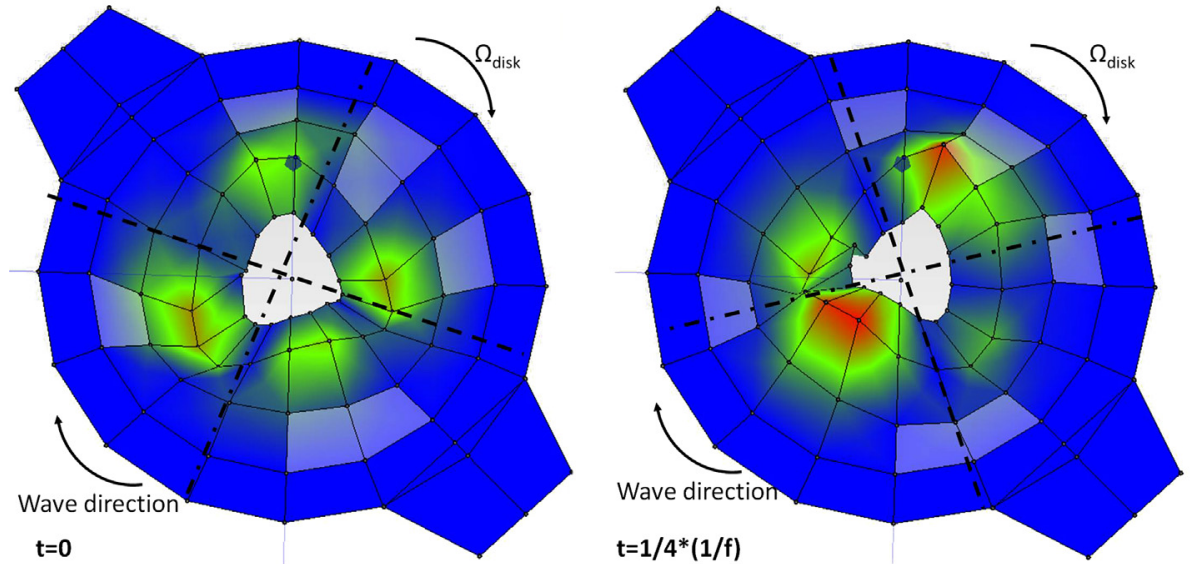

Fig. 20. Mode $n=2$ detected from the casing: $\Omega_{\text {disk }}=8 \mathrm{~Hz}$.
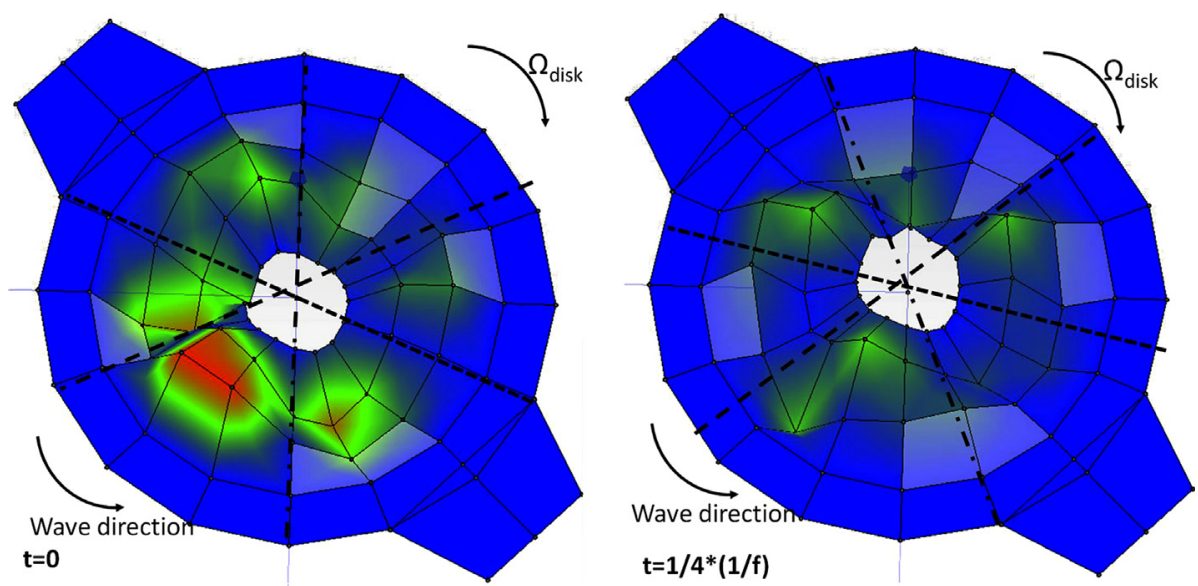

Fig. 21. Mode $n=-3$ detected from the casing: $\Omega_{\text {disk }}=8 \mathrm{~Hz}$.

In the shown ODS (Figs. 17-22), the modes $n= \pm 2$ and $n= \pm 3$ are clearly seen from the casing. Therefore, the conclusions of the analytical model have been validated in this section. One of the conditions for the applicability of the model is that the natural frequencies of the disk are well separated from the natural frequencies of the casing. For these modes, this condition has been accomplished as shown in Fig. 23, i.e. the response of the casing is low and the mode shape is well transmitted to the upper cover, since the casing is forced to move with this shape. 

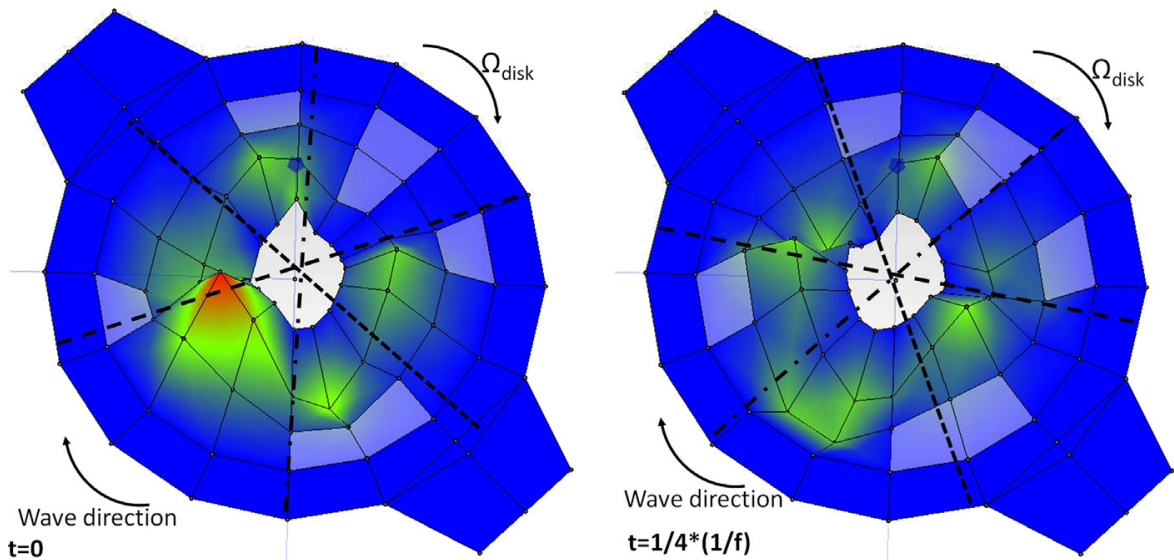

Fig. 22. Mode $n=3$ detected from the casing: $\Omega_{\text {disk }}=8 \mathrm{~Hz}$.

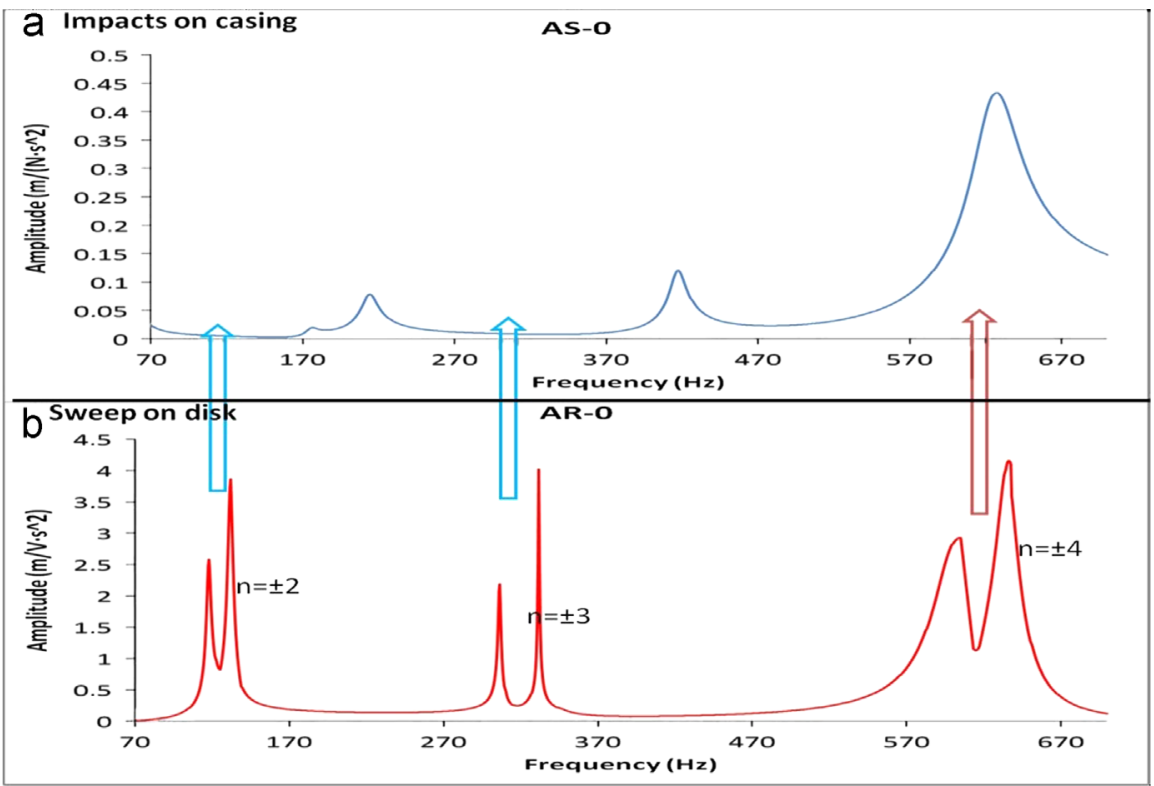

Fig. 23. (a) Response of the casing due to an impact on the casing, (b) response of the disk due to a sweep on the disk.

a

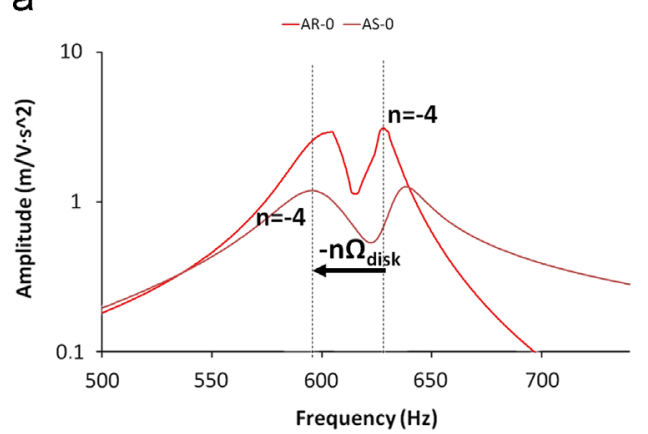

b

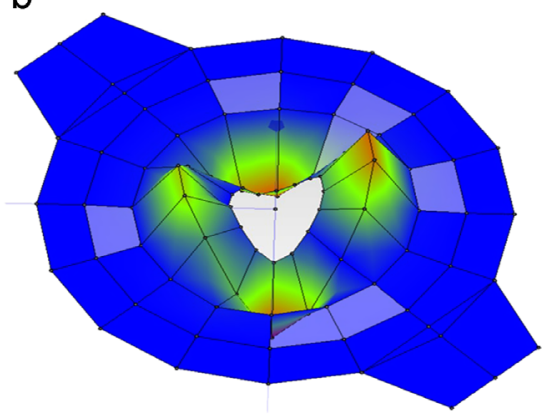

Fig. 24. Transmission of the mode $n=4$ and $n=-4$ to the upper cover $\left(\Omega_{\text {disk }}=8 \mathrm{~Hz}\right.$.): (a) peaks of AR- 0 and AS-0, (B) ODS of the casing at the peak detected in AS-0 for the mode $n=-4$.

Nevertheless for the pair of modes $n= \pm 4$ there is a high response of the casing. These modes are transmitted to the stationary frame as Fig. 24a shows, but the mode shape $n=-4$ of the disk is not recognizable from the upper cover (Fig. 24b). In this peak, the ODS is dominated by the mode of the casing located in this frequency band (Fig. 23a). 


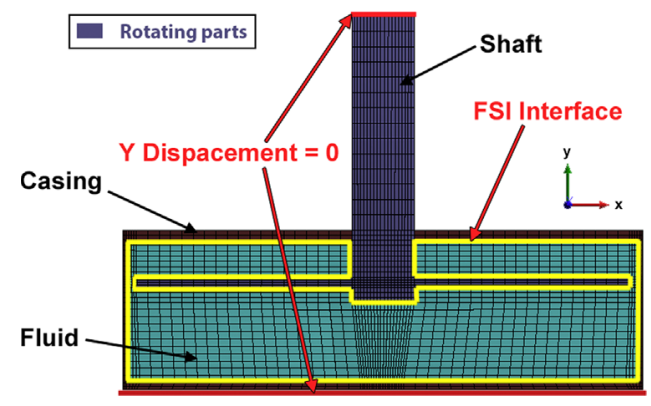

Fig. 25. Structural-acoustical FSI model.

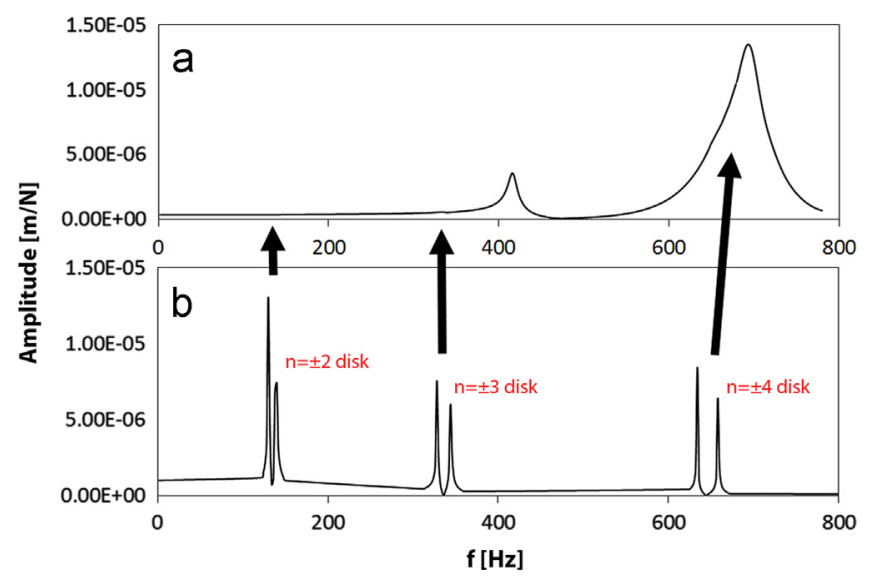

Fig. 26. Simulation response due to (a) sweep on the casing, (b) sweep on the disk.

\subsection{Modelling of the transmission with finite element model (FEM)}

A structural-acoustical FSI simulation $[23,38]$ has been performed in order to contrast the main conclusions of the experimental work, when the disk rotates in water. This type of simulation is based on the coupling of standard solid structures finite elements with an acoustical model that represents the fluid part. A model, has been built using Ansys Workbench ${ }^{\circledR}$ software (release 14.5). The model is constructed with hexahedral elements, using SOLID185 for the solid parts and FLUID30 for the liquid elements. Nodes of the solid parts in contact with the fluid are defined as a FSI (Fluid Structure Interaction) interface. The bottom of the tank is fixed in the y direction, as well as the top of the shaft. The mesh of the structural-acoustical FSI model including the applied boundary conditions is shown in Fig. 25.

The density of the fluid is fixed at $1000 \mathrm{~kg} / \mathrm{m}^{3}$ and the speed of sound was considered as $1430 \mathrm{~m} / \mathrm{s}$ according to the standard data for water (White [30]). The acoustic-structural coupling simulation assumes that the fluid is inviscid, irrotational and without mean flow.

A rotating speed is introduced on the rotating part (shaft + disk). The value of $\Omega_{\text {fluid }}$ (obtained from the CFD calculation) and $\Omega_{\text {disk }}$ are introduced in the FE acoustical model. Results are obtained from the stationary point of view.

A mesh sensitivity study was previously carried out to determine the optimal number of elements of the FEM model. The value of the natural frequency of the main mode shapes of the disk was selected as the variable to compare the element density of the mesh. The density of the mesh was changed in the axial and in the radial direction in order to consider all the possible parameters. The optimal mesh selected approximately had $7 \times 10^{4}$ elements.

In order to simulate the upper cover properly, the stiffness and the damping of this part are corrected using experimental modal analysis results (impacts on the casing as shown in Fig. 23a). Beta damping value [39] was the value calibrated for the modes of the casing. A harmonic analysis exciting the disk and the casing has been performed obtaining the following response characteristic (Fig. 26).

Both responses are viewed from the stationary frame [39]. Therefore, in this case for each diametrical mode of the disk (Fig. 26b), the first peak corresponds to the mode n-negative and the second peak to n-positive, on the contrary from the response detected from the rotating frame (Fig. 23b), due to the transmission between rotating and stationary frame (Eq. (22)). The response shown in this case is similar than the response of the experimental case, i.e. the modes $n= \pm 2$ and $n= \pm 3$ are well separated from the natural frequencies of the casing. There is a first peak from the casing which is detected experimentally (at about $230 \mathrm{~Hz}$ ) but not with the numerical FEM model. This peak corresponds to a mode which affects mainly the shaft system (Fig. 3) which is simplified in the simulation model (Fig. 25). 


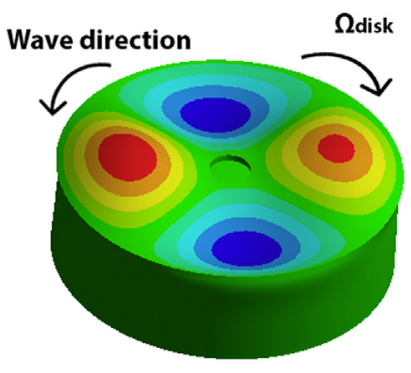

$\mathrm{T}=\mathbf{0}$

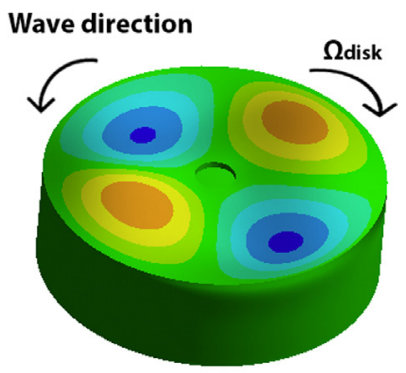

$T=(1 / 4)(1 / f)$

Fig. 27. Mode $n=-2$ detected from the casing: $\Omega_{\text {disk }}=8 \mathrm{~Hz}$ (FEM).
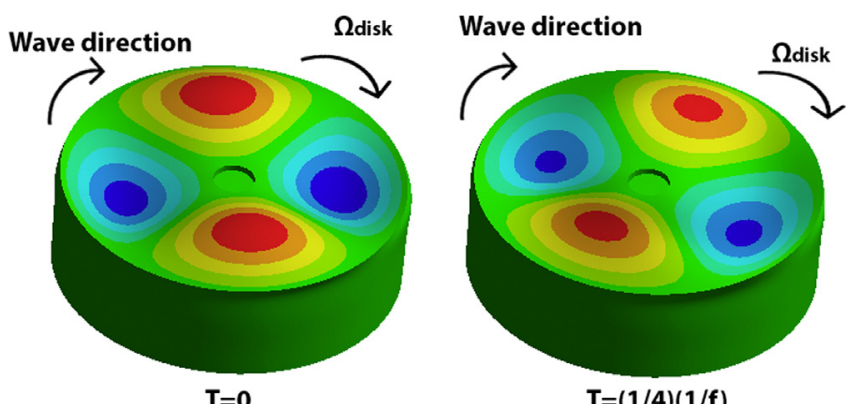

$T=(1 / 4)(1 / f)$

Fig. 28. Mode $n=+2$ detected from the casing: $\Omega_{\text {disk }}=8 \mathrm{~Hz}$ (FEM).

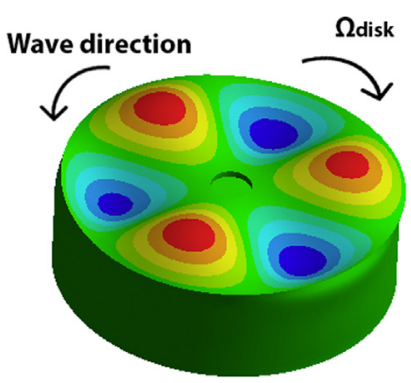

$\mathbf{T}=\mathbf{0}$

\section{Wave direction}

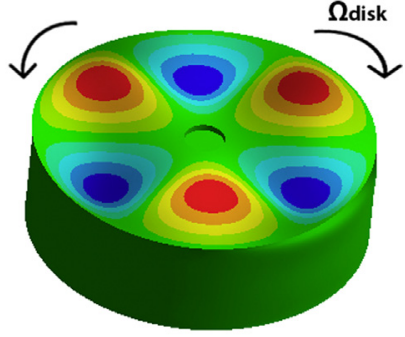

$\mathbf{T}=(\mathbf{1} / \mathbf{4})(\mathbf{1} / \mathbf{f})$

Fig. 29. Mode $n=-3$ detected from the casing: $\Omega_{\text {disk }}=8 \mathrm{~Hz}$ (FEM).
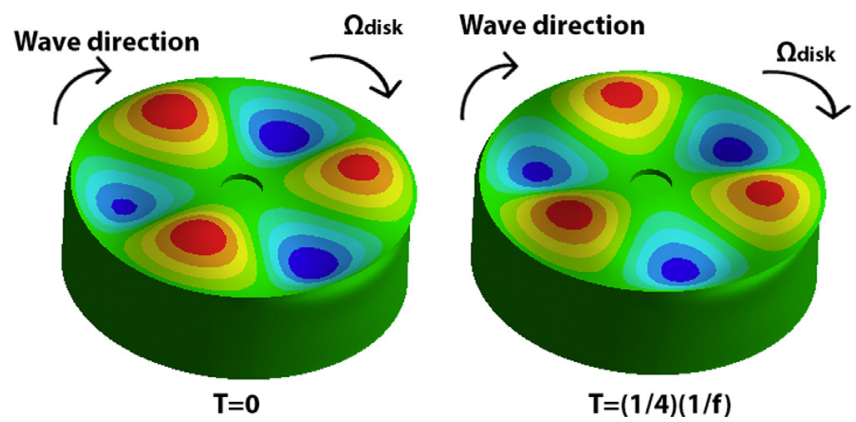

Fig. 30. Mode $n=+3$ detected from the casing: $\Omega_{\text {disk }}=8 \mathrm{~Hz}$ (FEM).

The modes $n= \pm 2$ and $n= \pm 3$ are clearly seen from the casing, when the disk is excited with a harmonic excitation (Fig. 27-30), as in the experimental results shown (Figs. 19-22).

Fig. 31 shows the case for the mode of the disk $n=-4$. As for the experimentation, the casing is dominated by this mode on this frequency band (Fig. 26). Therefore, the main conclusions from the analytical model that have been validated with experimentation, have been also validated with the numerical simulation. 


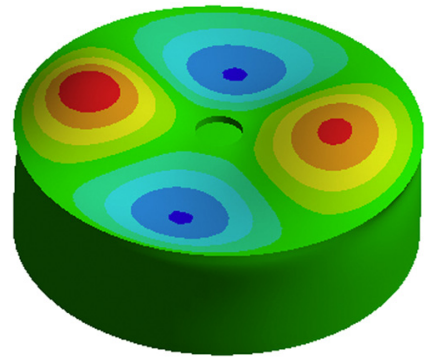

Fig. 31. Transmission of the mode $n=-4$ to the upper cover with FEM model. $\Omega_{\text {disk }}=8 \mathrm{~Hz}$.

\section{Conclusion}

In this paper, the detection of the natural frequencies and mode shapes of a rotating disk-like structure from the stationary frame has been studied experimentally and contrasted with an analytical model. The case of a disk that rotates in air (where the effects of the surrounding fluid can be neglected) has been analyzed in other researches but no studies have been found analyzing the same topic for a rotating disk-like structure surrounded by a heavy fluid (such as water). Only the diametrical modes, characterized by the number of nodal diameters $n$, which are the most relevant in case of turbomachinery components, are discussed here.

For this purpose, an experimental test rig has been developed. It consists of a submerged rotating disk inside a tank. The disk has been excited with a PZT from the rotating frame and its response measured from rotating and stationary frame in order to correlate the results of both reference frames.

Analyzing the rotating disk in air from the rotating frame, the effect of the rotation on the natural frequencies is very low for the tested rotating speeds of the disk $(0-8 \mathrm{~Hz})$. For one natural frequency in the rotating frame, simultaneously two natural frequencies are detected in the stationary frame shifted $\pm n \Omega_{\text {disk }}$ from the natural frequency detected in the rotating frame.

Analyzing the rotating disk in water from the rotating frame, the effect of rotation is very important. Each natural frequency in the non rotating case is transformed into two natural frequencies in the rotating case. If the disk is excited with a sweep, the two resonances occur at different time. The difference between both natural frequencies in the rotating case trends to increase when increasing $\Omega_{\text {disk }}$.

The lower natural frequency in the rotating frame, which is a travelling wave rotating in the same direction than the disk, is transmitted to a higher frequency in the stationary frame (frequency shift of $+n \Omega_{\text {disk }}$ ). The higher natural frequency in the rotating frame, which is a travelling wave rotating in counter direction than the disk, is transmitted to a lower frequency in the stationary frame (frequency shift of $-n \Omega_{\text {disk }}$ ). This has been proved using a time-frequency analysis with wavelets.

Not only the transmission of the natural frequencies has been studied, but also the transmission of the mode shapes. Experimental results have shown that a mode shape is transmitted from the rotating to the stationary frame if the response of the casing at the considered natural frequency is not relevant in comparison to the response of the disk. This has been checked with several types of sensors placed on the stationary frame, such as accelerometers, pressure sensors and a LDV (laser Doppler vibrometer).

The case of a disk natural frequency close to a high casing response band has been analyzed experimentally and numerically with a FEM model. Results show that in this case the peaks are detected from the casing but the mode shape is not transmitted and the response is dominated by the casing motion.

\section{References}

[1] E. Egusquiza, C. Valero, X. Huang, E. Jou, A. Guardo, C. Rodriguez, Failure investigation of a large pump-turbine runner, Eng. Fail. Anal. 23 (2012) 27-34.

[2] H. Tanaka, Vibration behavior and dynamic stress of runners of very high head reversible pump-turbines, Int. J. Fluid Mach. Syst. 4 (2011) 289-306.

[3] Y. Kubota, H. Ohashi, A study on the natural frequencies of hydraulic pumps, in: First ASME Joint International Conference on Nuclear Engineering, 1991 pp. 589-593.

[4] C.G. Rodriguez, E. Egusquiza, I.F. Santos, Frequencies in the vibration induced by the rotor stator interaction in a centrifugal pump turbine, J. Fluids Eng., Trans. ASME 129 (2007) 1428-1435.

[5] H. Ohashi, Case study of pump failure due to rotor-stator interaction, Int. J. Rotating Mach. 1 (1994) 53-60.

[6] X. Huang, Contribution to the Dynamic Response of Hydraulic Turbomachinery Components, CDIF, UPC, Barcelona, 2011.

[7] C.G. Rodriguez, E. Egusquiza, X. Escaler, Q.W. Liang, F. Avellan, Experimental investigation of added mass effects on a Francis turbine runner in still water, J. Fluids Struct. 22 (2006) 699-712.

[8] Q.W. Liang, C.G. Rodríguez, E. Egusquiza, X. Escaler, M. Farhat, F. Avellan, Numerical simulation of fluid added mass effect on a Francis turbine runner, Comput. Fluids 36 (2007) 1106-1118.

[9] X.X. Huang, E. Egusquiza, C. Valero, A. Presas, Dynamic behaviour of pump-turbine runner: from disk to prototype runner, in: IOP Conference Series: Materials Science and Engineering, 2013.

[10] A. Presas, C. Valero, X. Huang, E. Egusquiza, M. Farhat, F. Avellan, Analysis of the dynamic response of pump-turbine runners-Part I: Experiment, in: IOP Conference Series: Earth and Environmental Science, 2012.

[11] H. Lamb, R.V. Southwell, The vibrations of spinning discs, Proc. R. Soc. London 99 (1921) 272-280.

[12] W. Campbell, The protection of steam turbine disk wheels from axial vibration, Trans. ASME 46 (1924) $272-280$. 
[13] R.V. Southwell, On the free transverse vibrations of a uniform circular disc clamped at its centre and on the effects of rotation, Proc. R. Soc. London 101 (1922) 133-153.

[14] D.J. Ewins, The effects of detuning upon the forced vibrations of bladed disks, J. Sound Vib. 9 (1969) 65-79.

[15] L.E. El-Bayoumy, A.V. Srinivasan, Influence of mistuning on rotor-blade vibrations, AIAA J. 13 (1975) $460-464$.

[16] J.W. Heo, J. Chung, Vibration analysis of a flexible rotating disk with angular misalignment, J. Sound Vib. 274 (2004) $821-841$.

[17] H.F. Bauer, W. Eidel, Transverse vibration and stability of spinning circular plates of constant thickness and different boundary conditions, J. Sound Vib. 274 (2007) 877-895.

[18] L. Pust, L. Pesek, Vibration of circular bladed disk with imperfections, J. Bifurcation Chaos 10 (2011) $2893-2904$.

[19] H. Lamb, On the vibrations of an elastic plate in contact with water, Proc. R. Soc. London, Ser. A 98 (1921) 205-216.

[20] M.K. Kwak, Vibration of circular plates in contact with water, J. Appl. Mech. 58 (1991) 480-483.

[21] M Amabili, M.K. Kwak, Free vibrations of circular plates coupled with liquids: revising the Lamb problem, J. Fluids Struct. 10 (1996) $743-761$.

[22] E. Askari, K.-H. Jeong, M. Amabili, Hydroelastic vibration of circular plates immersed in a liquid-filled container with free surface, J. Sound Vib. 332 (2013) 3064-3085.

[23] D. Valentín, A. Presas, E. Egusquiza, C. Valero, Experimental study on the added mass and damping of a disk submerged in a partially fluid-filled tank with small radial confinement, J. Fluids Struct. 50 (2014) 1-17.

[24] J.A.N. Hengstler, Influence of the Fluid-Structure Interaction on the Vibrations of Structures, ETH Zurich, Zurich, 2013.

[25] A. Presas, E. Egusquiza, C. Valero, D. Valentin, U. Seidel, Feasibility of using PZT actuators to study the dynamic behavior of a rotating disk due to rotorstator interaction, Sensors 14 (2014) 11919-11942.

[26] T.K. Ahn, C.D.M. Mode Jr, Identification of a rotating disk, Exp. Mech. 38 (1998) 250-254.

[27] H. Mehdigholi, Forced Vibration of Rotating Discs and Interaction with Non-rotating Structures, Imperiall College of Science, Technology and Medicine, London, 1991.

[28] R. Belvins, Formulas for Natural Frequency and Mode Shape, Krieger Publishing Company, 1984.

[29] A.W. Leissa, Vibration of Plates, Goverment Printing Office, Washington (USA), 1969.

[30] F. White, Fluid Mechanics, McGraw-Hill, 1979.

[31] K. Singh, A.K. Mallik, Wave propagation and vibration response of a periodically supported pipe conveying fluid, J. Sound Vib. 54 (1977) 55-66.

[32] D.E. Newland, Wavelet analysis of vibration: Part 1-Theory, J. Vib. Acoust. 116 (1994) 409-416.

[33] C.K. Chui, Wavelet analysis and its applications, DTIC Document, 1995.

[34] Y. Wang, Z. He, Y. Zi, Enhancement of signal denoising and multiple fault signatures detecting in rotating machinery using dual-tree complex wavelet transform, Mech. Syst. Sig. Process. 24 (2010) 119-137.

[35] D.E. Newland, An Introduction to Random Vibrations, Spectral \& Wavelet Analysis, Courier Dover Publications, 2012.

[36] L. Cohen, Time-frequency Analysis, Prentice Hall PTR, Englewood Cliffs, NJ, 1995.

[37] F. Anger, P. Flandin, P. Gonçalves, O. Lemoine, in: C.a.R. University (Ed.), Time-Frequency Toolbox, C.a.R. University, 1996.

[38] C.G. Rodriguez, P. Flores, F.G. Pierart, L.R. Contzen, E. Egusquiza, Capability of structural-acoustical FSI numerical model to predict natural frequencies of submerged structures with nearby rigid surfaces, Comput. Fluids 64 (2012) 117-126.

[39] ANSYS, Ansys User's Manual 13.0., ANSYS, Canonsburg, USA, 2010. 